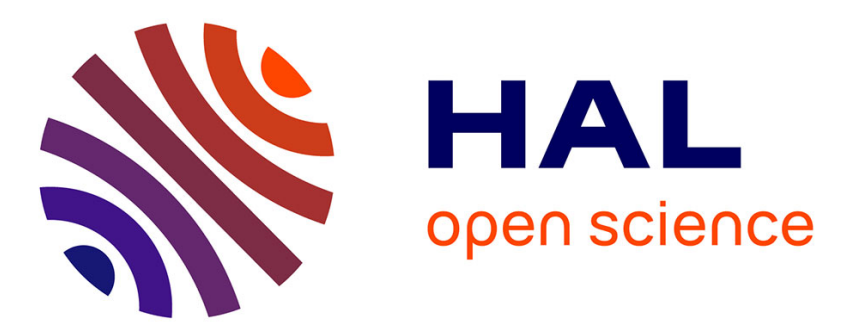

\title{
Gaussian semiflexible rings under angular and dihedral restrictions
}

Maxim Dolgushev, Thomas Guérin, Alexander Blumen, Olivier Benichou, Raphaël Voituriez

\section{- To cite this version:}

Maxim Dolgushev, Thomas Guérin, Alexander Blumen, Olivier Benichou, Raphaël Voituriez. Gaussian semiflexible rings under angular and dihedral restrictions. Journal of Chemical Physics, 2014, 141 (1), pp.014901 (1-13). 10.1063/1.4885445 . hal-01063638

\section{HAL Id: hal-01063638 \\ https://hal.science/hal-01063638}

Submitted on 25 Feb 2015

HAL is a multi-disciplinary open access archive for the deposit and dissemination of scientific research documents, whether they are published or not. The documents may come from teaching and research institutions in France or abroad, or from public or private research centers.
L'archive ouverte pluridisciplinaire HAL, est destinée au dépôt et à la diffusion de documents scientifiques de niveau recherche, publiés ou non, émanant des établissements d'enseignement et de recherche français ou étrangers, des laboratoires publics ou privés.

\section{다(1)(2)}

Distributed under a Creative Commons Attribution - ShareAlikel 4.0 International 


\title{
Gaussian semiflexible rings under angular and dihedral restrictions
}

\author{
Maxim Dolgushev, ${ }^{1, a)}$ Thomas Guérin, ${ }^{2,3}$ Alexander Blumen, ${ }^{1}$ Olivier Bénichou, ${ }^{2}$ \\ and Raphaël Voituriez ${ }^{2}$ \\ ${ }^{1}$ Theoretical Polymer Physics, University of Freiburg, Hermann-Herder-Str. 3, 79104 Freiburg, Germany \\ ${ }^{2}$ Laboratoire de Physique Théorique de la Matière Condensée, Centre National de la Recherche Scientifique, \\ Université Pierre et Marie Curie, 4 Place Jussieu, 75005 Paris, France \\ ${ }^{3}$ Laboratoire Ondes et Matière d'Aquitaine, University of Bordeaux, Unité Mixte de Recherche 5798, \\ Centre National de la Recherche Scientifique, 33400 Talence, France
}

Semiflexible polymer rings whose bonds obey both angular and dihedral restrictions [M. Dolgushev and A. Blumen, J. Chem. Phys. 138, 204902 (2013)], are treated under exact closure constraints. This allows us to obtain semianalytic results for their dynamics, based on sets of Langevin equations. The dihedral restrictions clearly manifest themselves in the behavior of the mean-square monomer displacement. The determination of the equilibrium ring conformations shows that the dihedral constraints influence the ring curvature, leading to compact folded structures. The method for imposing such constraints in Gaussian systems is very general and it allows to account for heterogeneous (sitedependent) restrictions. We show it by considering rings in which one site differs from the others.

\section{INTRODUCTION}

Semiflexible polymers continue to be very interesting subjects of study, ${ }^{1-15}$ since many biological macromolecules, e.g., actin and DNA have high persistence lengths. ${ }^{16-18}$ A widespread way of modeling the semiflexibility of chains consists in fixing the angles between nearest-neighbors' (NN) bonds, under the additional assumption that rotations around the axis given by each bond are free. Under these assumptions the basic properties of linear semiflexible polymers can be obtained in a straightforward way, which also explains the success of the model. ${ }^{1,2,4,7}$ Furthermore, these assumptions can also be used to treat vastly more general, semiflexible treelike (loop-less) polymers (STP) with arbitrary structure. ${ }^{9,19-23}$ The model proceeds from a coarse-grained picture of Gaussian generalized structures (GGS) ${ }^{24}$ (which involves beads and springs) into which the restrictions over bonds are implemented.

From a chemical perspective, the bonds in a polymer cannot rotate freely ${ }^{25}$ and one has to account for hindered bond rotations, especially when treating macromolecules with high persistence length. ${ }^{26,27}$ Such hindered rotations were recently introduced into the GGS scheme, as exemplified for linear chains, in Ref. 28. Figure 1 illustrates the angles entering the model: $\theta$ denotes the angle between oriented nearest-neighbor bonds, say $\mathbf{d}_{1}$ and $\mathbf{d}_{2}$ or $\mathbf{d}_{2}$ and $\mathbf{d}_{3}, \phi$ is the dihedral angle between the planes spanned by the bond pairs $\left(\mathbf{d}_{1}, \mathbf{d}_{2}\right)$ and $\left(\mathbf{d}_{2}, \mathbf{d}_{3}\right)$. In Ref. 28, dihedral restrictions (DR) were implemented by fixing the average values of the scalar products of the corresponding bonds. In this way, the constraints do not change the Gaussian character of the model, since they can be incorporated into the covariance matrix of the multivariate (Gaussian) distribution of bonds.

\footnotetext{
a)Electronic mail: dolgushev@ physik.uni-freiburg.de
}

Now, STP are devoid of loops, and hence rings do not belong to the STP class. On the other hand, many proteins are topologically equivalent to rings, ${ }^{29}$ and the question arises how to model them. ${ }^{30-33}$ We recall that for rings, an additional, strict closure constraint holds: ${ }^{33,34}$ If we represent a ring through a series of bond vectors, consecutively connected in head-to-tail conformations, the sum of all these vectors has to vanish (a holonomic constraint). In this way, and different from an open chain, the bond vectors of a ring are not anymore independent variables; a ring has less degrees of freedom. ${ }^{35}$

Remarkably, however, in the case of Gaussian bond distributions for chains, such a holonomic constraint leads to a reduced set of independent bond variables for the ring formed by closing the chain, variables which still remain Gaussian. This remarkable fact is fundamental for us here. Now, under freely rotating conditions one can (tediously) determine this new bond distribution for the ring by algebraically getting rid of the dependent bond variables. ${ }^{33}$ Here, we highlight more powerful methods, such as introducing a penalty function for imperfect closure, or, even more general, determining the new distribution of bonds by employing mathematical methods such as given in Ref. 36. Recently, these methods have been used in treating non-Markovian diffusion-limited reactions. ${ }^{37-39}$ There, ${ }^{37-39}$ as well as in this work, the fundamental approach is the Gaussian statistics of bonds.

In this paper, we put forward the penalty function method to study semiflexible polymer rings under angular and dihedral restrictions. The method gets rid of many problems involved in treating loops, especially in what introducing closure conditions are concerned. Moreover, the method allows us to obtain results to a great extent analytically, here in particular for the dynamical matrix and for its eigenvalues. Based on these results, we investigate static and dynamical properties of semiflexible rings, focusing on their bond-bond correlations, on their gyration radius, and on the monomers' 


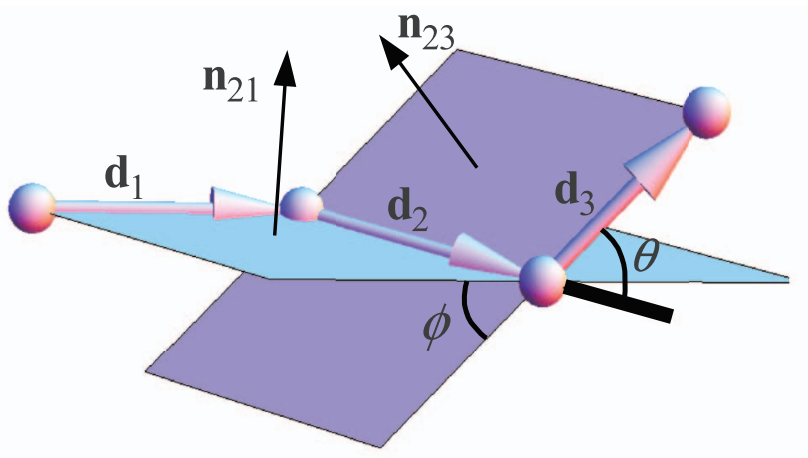

FIG. 1. Schematic picture of three consecutively connected bonds $\mathbf{d}_{1}, \mathbf{d}_{2}$, and $\mathbf{d}_{3}$. In the figure, $\theta$ denotes the angle between neighboring bonds (here, $\mathbf{d}_{2}$ and $\left.\mathbf{d}_{3}\right), \phi$ is the dihedral angle between the planes spanned by the pairs $\left(\mathbf{d}_{1}, \mathbf{d}_{2}\right)$ and $\left(\mathbf{d}_{2}, \mathbf{d}_{3}\right), \mathbf{n}_{21}$ and $\mathbf{n}_{23}$ are the normal vectors to the planes.

mean-square displacements (MSD). We recall that the gyration radius can be determined experimentally using standard scattering methods ${ }^{40}$ and that the other quantities are experimentally accessible through modern atomic-force microscopy techniques. ${ }^{41-43}$ We evaluate these quantities for rings under DR and compare them to those for rings in the absence of DR. As we proceed to show, the DR influence very much the properties of rings: While rings including DR often display a winding structure, rings without DR do not show this feature. This fact leaves its fingerprints on the other quantities considered here, namely, on the gyration radius and on the monomers' mean-square displacement.

In homogeneous semiflexible rings, the stiffness conditions are identical at every site. This is distinct from nonhomogeneous (heterogeneous) rings, where the conditions may differ from site to site. Here, the penalty function method allows us to treat both homogeneous and heterogeneous rings with DR, the latter exemplified by a ring in which the stiffness conditions at one site differ from the rest. This difference arises naturally when monitoring the closing (cyclization) of a homogeneous chain, as depicted in Fig. 2. As we proceed to show, homogeneous and nonhomogeneous rings have quite distinct static and dynamic behaviors.

The paper is structured as follows: We start in Sec. II by recalling how angular and dihedral restrictions are implemented, ${ }^{28}$ and introduce the penalty function method for the closure of rings. Based on this method we investigate in Sec. III the dynamics of homogeneous rings. Section IV is devoted to the study of particular nonhomogeneous rings, where we determine their dynamical properties based on the method of Sec. II; here, we also point out the connection to the general method of Ref. 36. In Sec. V, we analyze the obtained results for both types of rings and focus on the special role of the DR. The article ends with our conclusions, whereas mathematical details as well as some particular limiting cases of our models are relegated to Appendixes A-C.

\section{MODELING ANGULAR AND DIHEDRAL RESTRICTIONS}

Here, we briefly summarize the ideas of Ref. 28 for modeling local angular and dihedral constraints for chains and extend them to homogeneous rings. (a)

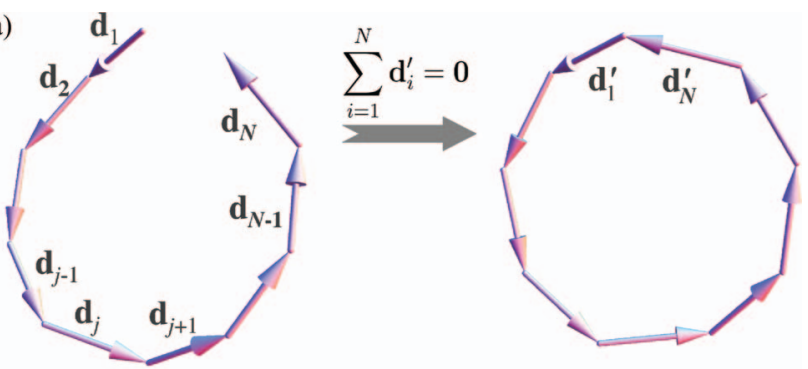

(b)

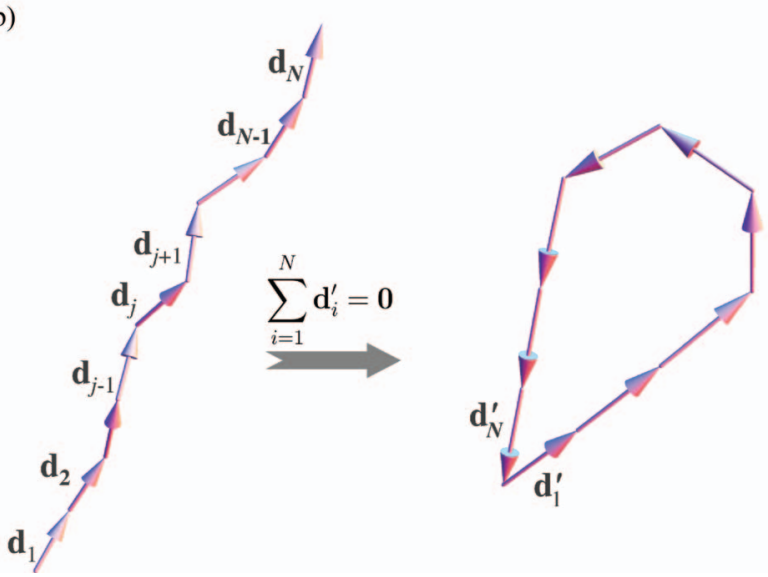

FIG. 2. Schematic cyclization scenarios. One starts from linear chains represented by bonds $\left\{\mathbf{d}_{a}\right\}$, from which rings consisting of $N$ bonds $\left\{\mathbf{d}_{a}^{\prime}\right\}$ are constructed. The upper box (a) displays the construction of a homogeneous semiflexible ring (bond variables $\left\{\mathbf{d}_{a}^{\prime}\right\}$ ) by formally imposing the closure condition, $\sum_{i=1}^{N} \mathbf{d}_{i}^{\prime}=0$, while the $\left\{\mathbf{d}_{a}^{\prime}\right\}$ behave homogeneously, e.g., $\left\langle\mathbf{d}_{1}^{\prime} \cdot \mathbf{d}_{N}^{\prime}\right\rangle$ $=\left\langle\mathbf{d}_{j}^{\prime} \cdot \mathbf{d}_{j \pm 1}^{\prime}\right\rangle$ and $\left\langle\mathbf{d}_{1}^{\prime} \cdot \mathbf{d}_{N-1}^{\prime}\right\rangle=\left\langle\mathbf{d}_{2}^{\prime} \cdot \mathbf{d}_{N}^{\prime}\right\rangle=\left\langle\mathbf{d}_{j+1}^{\prime} \cdot \mathbf{d}_{j-1}^{\prime}\right\rangle$. The lower box (b) shows the construction of a nonhomogeneous semiflexible ring, without imposing any additional restrictions on the terminal bonds of the chain, see text for details.

Consider locally three oriented bonds, say $\mathbf{d}_{1}, \mathbf{d}_{2}$, and $\mathbf{d}_{3}$, which are consecutively connected head-to-tail, as schematically shown in Fig. 1. Here, $\theta$ denotes the angle between NN bonds, say $\mathbf{d}_{2}$ and $\mathbf{d}_{3}$. Furthermore, $\phi$ denotes the dihedral angle between the planes spanned by consecutive NN bond pairs, say $\left(\mathbf{d}_{1}, \mathbf{d}_{2}\right)$ and $\left(\mathbf{d}_{2}, \mathbf{d}_{3}\right)$, see Fig. 1 . Then $\phi=0$ is related to a flat, zigzag bond configuration. ${ }^{25}$ Setting $\mathbf{n}_{21} \equiv \mathbf{d}_{2} \times \mathbf{d}_{1}$ and $\mathbf{n}_{23} \equiv \mathbf{d}_{2} \times \mathbf{d}_{3}, \mathbf{n}_{21}$ and $\mathbf{n}_{23}$ are orthogonal to the respective planes and $\phi$ is the angle between $\mathbf{n}_{21}$ and $\mathbf{n}_{23}$.

Now, semiflexibility is modeled by imposing constraints on the average scalar products of the bonds. First, we constrain the mean-square length of each bond, by setting

$$
\left\langle\mathbf{d}_{a} \cdot \mathbf{d}_{a}\right\rangle=l^{2} .
$$

Second, we restrict the average angle between NN bonds, say $\mathbf{d}_{a}$ and $\mathbf{d}_{b}$,

$$
\left\langle\mathbf{d}_{a} \cdot \mathbf{d}_{b}\right\rangle=l^{2} \widetilde{q}_{1},
$$

where the stiffness parameter $\widetilde{q}_{1}$ is related to $\langle\cos \theta\rangle$. Third, we constrain the next-nearest-neighbor (NNN) bonds, say $\mathbf{d}_{a}$ and $\mathbf{d}_{c}$,

$$
\left\langle\mathbf{d}_{a} \cdot \mathbf{d}_{c}\right\rangle=l^{2} \widetilde{q}_{2},
$$


where $\widetilde{q}_{2}=\widetilde{q}_{1}^{2}+\widetilde{p}\left(1-\widetilde{q}_{1}^{2}\right)$ with $\widetilde{p}$ related to $\langle\cos \phi\rangle .{ }^{28}$ In this way, through Eqs. (1)-(3), we account both for the angular and for the dihedral restrictions, which depend now on the stiffness parameters $\widetilde{q}_{1}$ and $\widetilde{q}_{2}$. We note that the parameters $\widetilde{q}_{1}$ and $\widetilde{q}_{2}$ are introduced in an averaged way, so that they may reflect different distributions of the angular restrictions and are not related to fixed values of the angles $\theta$ and $\phi$, see, e.g., Ref. 25.

Furthermore, as has been shown in Ref. 28 the potential energy of the chain under the local constraints of Eqs. (1)-(3) has the form

$$
V\left(\left\{\mathbf{d}_{a}\right\}\right)=\frac{K}{2} \sum_{a, b} \widetilde{W}_{a b} \mathbf{d}_{a} \cdot \mathbf{d}_{b},
$$

where $K=3 k_{B} T / l^{2}$ denotes the spring constant, $k_{B}$ is the Boltzmann constant, $T$ is the temperature, and the sum runs over all bonds. Moreover, the matrix $\widetilde{\mathbf{W}}=\left(\widetilde{W}_{a b}\right)$ is sparse and it has the following form: ${ }^{28}$

$$
\widetilde{\mathbf{W}}=\left(\begin{array}{cccccccc}
\alpha^{\prime} & \beta^{\prime} & \gamma & 0 & \ldots & 0 & 0 & 0 \\
\beta^{\prime} & \alpha^{\prime \prime} & \beta & \gamma & 0 & \ldots & 0 & 0 \\
\gamma & \beta & \alpha & \beta & \gamma & 0 & \ldots & 0 \\
0 & \gamma & \beta & \ddots & \ddots & \ddots & \ddots & \vdots \\
\vdots & \ddots & \ddots & \ddots & \ddots & \beta & \gamma & 0 \\
0 & \ldots & 0 & \gamma & \beta & \alpha & \beta & \gamma \\
0 & 0 & \ldots & 0 & \gamma & \beta & \alpha^{\prime \prime} & \beta^{\prime} \\
0 & 0 & 0 & \ldots & 0 & \gamma & \beta^{\prime} & \alpha^{\prime}
\end{array}\right) .
$$

Thus, for a chain consisting of consecutively numbered bonds $\mathbf{d}_{1}, \mathbf{d}_{2}, \ldots, \mathbf{d}_{N}$, the $N \times N$ matrix $\widetilde{\mathbf{W}}$ is a symmetric, pentadiagonal matrix. ${ }^{28}$ In other words, the nonvanishing offdiagonal elements of $\widetilde{\mathbf{W}}$ are related only to the $\mathrm{NN}$ and to the NNN neighbors. The explicit values of $\alpha, \beta, \gamma, \alpha^{\prime}, \alpha^{\prime \prime}$, and $\beta^{\prime}$ are given as a function of $\widetilde{q}_{1}$ and $\widetilde{q}_{2}$ in Appendix A. One may note the difference between the parameters $\alpha^{\prime}, \alpha^{\prime \prime}$, and $\beta^{\prime}$ corresponding to peripheral bonds and the values $\alpha$ and $\beta$ for the internal bonds. ${ }^{28}$

Now, having in mind the symmetry of a homogeneous ring (in which all bonds are internal) we infer that the corresponding matrix $\mathbf{W}$ has the general form

$$
\mathbf{W}=\left(\begin{array}{cccccccc}
\alpha & \beta & \gamma & 0 & \ldots & 0 & \gamma & \beta \\
\beta & \alpha & \beta & \gamma & 0 & \ldots & 0 & \gamma \\
\gamma & \beta & \alpha & \beta & \gamma & 0 & \ldots & 0 \\
0 & \gamma & \beta & \ddots & \ddots & \ddots & \ddots & \vdots \\
\vdots & \ddots & \ddots & \ddots & \ddots & \beta & \gamma & 0 \\
0 & \ldots & 0 & \gamma & \beta & \alpha & \beta & \gamma \\
\gamma & 0 & \ldots & 0 & \gamma & \beta & \alpha & \beta \\
\beta & \gamma & 0 & \ldots & 0 & \gamma & \beta & \alpha
\end{array}\right),
$$

as can also be obtained from the maximum entropy principle. ${ }^{44,45}$
The knowledge of the matrix $\mathbf{W}$ together with the fact that for rings the closure condition

$$
\sum_{i=1}^{N} \mathbf{d}_{i}=\mathbf{0}
$$

holds, leads to the distribution of the $\left\{\mathbf{d}_{a}\right\}$, which can be formally expressed as

$$
P\left(\left\{\mathbf{d}_{a}\right\}\right)=\frac{1}{Z} \exp \left(-\frac{3}{2 l^{2}} \sum_{a, b} W_{a b} \mathbf{d}_{a} \cdot \mathbf{d}_{b}\right) \delta\left(\mathbf{d}_{1}+\cdots+\mathbf{d}_{N}\right),
$$

where $\delta(x)$ denotes the Dirac delta-function. Moreover, recalling the realization of $\delta(x)$ in the limit of small $\epsilon$ through the function $e^{-x^{2} / \epsilon^{2}} / \sqrt{\epsilon^{2} \pi}$ we insert the latter into Eq. (8), obtaining

$$
\begin{aligned}
& P\left(\left\{\mathbf{d}_{a}\right\}\right) \\
& \quad=\frac{1}{Z^{\prime}} \exp \left[-\frac{3}{2 l^{2}}\left(\sum_{a, b} W_{a b} \mathbf{d}_{a} \cdot \mathbf{d}_{b}+\frac{1}{\epsilon^{2}}\left(\mathbf{d}_{1}+\cdots+\mathbf{d}_{N}\right)^{2}\right)\right],
\end{aligned}
$$

where $0<\epsilon \ll 1$. Equation (9) reads thus

$$
P\left(\left\{\mathbf{d}_{a}\right\}\right)=\frac{1}{Z^{\prime}} \exp \left(-\frac{3}{2 l^{2}} \sum_{a, b} \widehat{W}_{a b} \mathbf{d}_{a} \cdot \mathbf{d}_{b}\right),
$$

where the matrix $\widehat{\mathbf{W}}=\left\{\widehat{W}_{a b}\right\}$ is given by

$$
\widehat{\mathbf{W}}=\mathbf{W}+\frac{1}{\epsilon^{2}} \mathbf{L}
$$

with $(\mathbf{L})_{i j}=1$. As we proceed to show, it turns out to be very convenient to work first with Eq. (11) and to take later the limit $\epsilon \rightarrow 0^{+}$, by which the results become independent of $\epsilon$. To be more specific: The term proportional to $1 / \epsilon^{2}$ in Eq. (9) acts as an energetic penalty for chains that did not yet completely close to a ring. In this way, we avoid to use Eq. (7) in a direct manner, which necessitates expressing one bond as a linear combination of the others (see Ref. 33); this is algebraically very cumbersome and breaks the symmetry of the problem.

\section{HOMOGENEOUS RINGS}

\section{A. Relation of the stiffness parameters $q_{1}$ and $q_{2}$ to the elements of $W$}

Here, we consider homogeneous rings whose bonds $\left\{\mathbf{d}_{a}\right\}$ follow the distribution Eq. (10). We note, furthermore, that letting $\epsilon$ be small $(\epsilon>0)$, but having $\epsilon \neq 0$, keeps the set of vectors $\left\{\mathbf{d}_{a}\right\}$ independent. Due to this fact the average values of $\mathbf{d}_{a} \cdot \mathbf{d}_{b}$ can be obtained by integrating them with respect to the Gaussian form Eq. (10) and are given through ${ }^{28}$

$$
\left\langle\mathbf{d}_{a} \cdot \mathbf{d}_{b}\right\rangle=l^{2}\left(\widehat{\mathbf{W}}^{-1}\right)_{a b} .
$$

The task consists now in determining the inverse of $\widehat{\mathbf{W}}$, which can be found through the diagonalization of $\widehat{\mathbf{W}}=\mathbf{W}$ $+\left(1 / \epsilon^{2}\right) \mathbf{L}$. First, we note that due to its symmetry, the 
periodically symmetric matrix $\mathbf{W}$, Eq. (6), can be readily diagonalized by means of the discrete Fourier transform ${ }^{46}$

$$
C_{j k}=\frac{1}{\sqrt{N}} \exp \left(\mathrm{i} \frac{2 \pi k j}{N}\right) .
$$

The ensuing eigenvalues of $\mathbf{W}$ are

$$
\lambda_{k}=\alpha+2 \beta \cos \left(\frac{2 \pi k}{N}\right)+2 \gamma \cos \left(\frac{4 \pi k}{N}\right),
$$

where $k$ runs from 1 to $N$. Furthermore, given that $\sum_{j} C_{j k}$ $=\sqrt{N} \delta_{k N}$, the matrix $\mathbf{C}$ also diagonalizes $\mathbf{L}$, leading to the eigenvalues $(0, \ldots, 0, N)$. Thus, the eigenvalues of $\widehat{\mathbf{W}}$ are $\widehat{\lambda}_{i}=\lambda_{i}$ for $i=1, \ldots,(N-1)$ and $\widehat{\lambda}_{N}=\lambda_{N}+N / \epsilon^{2}$. In this way, we obtain $\mathbf{C}^{-1} \widehat{\mathbf{W}} \mathbf{C}=\widehat{\boldsymbol{\Lambda}}$, where $\widehat{\boldsymbol{\Lambda}}=\operatorname{Diag}\left(\widehat{\lambda}_{1}, \ldots, \widehat{\lambda}_{N}\right)$ is a diagonal matrix. Then, based on $\widehat{\boldsymbol{\Lambda}}$, we get

$$
\widehat{\mathbf{W}}^{-1}=\mathbf{C} \widehat{\boldsymbol{\Lambda}}^{-1} \mathbf{C}^{-1} \text {. }
$$

Hence, inserting Eq. (13) into Eq. (15) we obtain

$$
\begin{aligned}
\left(\widehat{\mathbf{W}}^{-1}\right)_{a b}= & \frac{1}{N\left(\lambda_{N}+N / \epsilon^{2}\right)} \\
& +\frac{1}{N} \sum_{k=1}^{N-1} \frac{\cos [2 \pi k(a-b) / N]}{\alpha+2 \beta \cos \left(\frac{2 \pi k}{N}\right)+2 \gamma \cos \left(\frac{4 \pi k}{N}\right)} .
\end{aligned}
$$

Finally, using Eq. (12) and taking the limit $\epsilon \rightarrow 0$ we are led to

$$
\left\langle\mathbf{d}_{a} \cdot \mathbf{d}_{b}\right\rangle=\frac{l^{2}}{N} \sum_{k=1}^{N-1} \frac{\cos [2 \pi k(a-b) / N]}{\alpha+2 \beta \cos \left(\frac{2 \pi k}{N}\right)+2 \gamma \cos \left(\frac{4 \pi k}{N}\right)} .
$$

Now, the parameters $\alpha, \beta$, and $\gamma$ are connected to the local constraints, Eqs. (1)-(3). To see this we recall Eq. (1), which reads now explicitly, say for the $a$ th bond $(a=1, \ldots$, $N)$

$$
l^{2}=\left\langle\mathbf{d}_{a} \cdot \mathbf{d}_{a}\right\rangle=\frac{l^{2}}{N} \sum_{k=1}^{N-1} \frac{1}{\alpha+2 \beta \cos \left(\frac{2 \pi k}{N}\right)+2 \gamma \cos \left(\frac{4 \pi k}{N}\right)} .
$$

It is worth to notice that the rhs of Eq. (18) is (as it should be) independent of the bond number $a$. Second, we view $\left\langle\mathbf{d}_{a}\right.$. $\left.\mathbf{d}_{a+1}\right\rangle$ and $\left\langle\mathbf{d}_{a} \cdot \mathbf{d}_{a+2}\right\rangle$ in the spirit of Eqs. (2) and (3), namely, as reflecting the angular $(\mathrm{NN})$ and the dihedral $(\mathrm{NNN})$ restrictions. Hence, based on Eq. (17), it follows:

$$
\begin{aligned}
l^{2} q_{1} & =\left\langle\mathbf{d}_{a} \cdot \mathbf{d}_{a+1}\right\rangle=\left\langle\mathbf{d}_{N} \cdot \mathbf{d}_{1}\right\rangle \\
& =\frac{l^{2}}{N} \sum_{k=1}^{N-1} \frac{\cos (2 \pi k / N)}{\alpha+2 \beta \cos \left(\frac{2 \pi k}{N}\right)+2 \gamma \cos \left(\frac{4 \pi k}{N}\right)}
\end{aligned}
$$

and

$$
\begin{aligned}
l^{2} q_{2} & =\left\langle\mathbf{d}_{a} \cdot \mathbf{d}_{a+2}\right\rangle=\left\langle\mathbf{d}_{N-1} \cdot \mathbf{d}_{1}\right\rangle=\left\langle\mathbf{d}_{N} \cdot \mathbf{d}_{2}\right\rangle \\
& =\frac{l^{2}}{N} \sum_{k=1}^{N-1} \frac{\cos (4 \pi k / N)}{\alpha+2 \beta \cos \left(\frac{2 \pi k}{N}\right)+2 \gamma \cos \left(\frac{4 \pi k}{N}\right)} .
\end{aligned}
$$

Here, again the rhs of Eqs. (19) and (20) are independent of the bond number $a$. The parameters $q_{1}$ and $q_{2}$ for the homogeneous ring can now be expressed in terms of $\alpha, \beta$, and $\gamma$ (and also of $\widetilde{q}_{1}, \widetilde{q}_{2}$, and $N$ ), say by taking the quotients of the rhs of Eqs. (19) and (18) or (20) and (18), respectively. Also the reverse is true: The set of Eqs. (18)-(20) allows to determine $\alpha, \beta$, and $\gamma$ as functions of $l^{2}, q_{1}$, and $q_{2}$. The way of doing so is exemplified in the supplementary material. ${ }^{47}$

\section{B. Dynamics}

In order to determine the dynamical properties of homogeneous rings, we invoke the corresponding Langevin equations (LE). Denoting the positions of the ring's monomers by $\left\{\mathbf{r}_{i}=\left(x_{i}, y_{i}, z_{i}\right)\right\}$, the LE, e.g., for the $y$-component of the $j$ th monomer reads ${ }^{24,48,49}$

$$
\zeta \frac{\partial}{\partial t} y_{j}(t)+\frac{\partial}{\partial y_{j}} V\left(\left\{\mathbf{r}_{i}\right\}\right)=f_{j}(t) .
$$

Here, $V\left(\left\{\mathbf{r}_{i}\right\}\right)$ is the potential energy written as function of the set of all $\left\{\mathbf{r}_{i}\right\}$ and $f_{j}$ is the $y$-component of the usual fluctuating Gaussian force acting on the $j$ th bead, for which $\left\langle f_{j}(t)\right\rangle=0$ and $\left\langle f_{j}(t) f_{k}\left(t^{\prime}\right)\right\rangle=2 k_{B} T \zeta \delta_{j k} \delta\left(t-t^{\prime}\right)$ hold.

In order to determine the potential as a function of the $\mathbf{r}_{i}$, we have to reformulate the distribution $P\left(\left\{\mathbf{d}_{a}\right\}\right)$, Eq. (10), as a function of the bead variables $\left\{\mathbf{r}_{i}\right\}$. The transformation from bond to bead variables is given by the transposed matrix $\mathbf{G}^{T}$ of the incidence matrix $\mathbf{G}=\left(G_{i a}\right)^{21,50}$

$$
\mathbf{d}_{a}=\sum_{k=1}^{N}\left(\mathbf{G}^{T}\right)_{a k} \mathbf{r}_{k}
$$

where $\mathbf{G}$ reads

$$
\mathbf{G}=\left(\begin{array}{ccccc}
-1 & 0 & \ldots & 0 & 1 \\
1 & -1 & 0 & \ldots & 0 \\
0 & \ddots & \ddots & \ddots & \vdots \\
\vdots & \ddots & \ddots & \ddots & 0 \\
0 & \ldots & 0 & 1 & -1
\end{array}\right) .
$$

Distinct from linear chains and from general treelike structures, for rings $\mathbf{G}$ is a square $N \times N$ matrix, since for rings the number of beads is equal to the number of bonds. Substitution of Eq. (22) into Eq. (10) leads to

$$
P\left(\left\{\mathbf{r}_{i}\right\}\right)=\frac{1}{Z^{\prime}} \exp \left(-\frac{3}{2 l^{2}} \sum_{k, n}\left(\mathbf{G} \widehat{\mathbf{W}} \mathbf{G}^{T}\right)_{k n} \mathbf{r}_{k} \cdot \mathbf{r}_{n}\right) .
$$

Multiplying $\mathbf{G}$ of Eq. (23) with $\mathbf{L} / \epsilon^{2}$ of Eq. (11) leads to a matrix of zeros. Hence, from Eq. (11) we have $\mathbf{G} \widehat{\mathbf{W}} \mathbf{G}^{T}$ $=\mathbf{G W G}^{T}$. Thus, the distribution $P$ reads

$$
P\left(\left\{\mathbf{r}_{i}\right\}\right)=\frac{1}{Z^{\prime}} \exp \left(-\frac{3}{2 l^{2}} \sum_{k, n}\left(\mathbf{G W G}^{T}\right)_{k n} \mathbf{r}_{k} \cdot \mathbf{r}_{n}\right) .
$$

Associating Eq. (25) with the Boltzmann distribution, we hence infer the existence of an effective potential for the ring of the form

$$
V\left(\left\{\mathbf{r}_{i}\right\}\right)=\frac{K}{2} \sum_{k, n}\left(\mathbf{G W G}^{T}\right)_{k n} \mathbf{r}_{k} \cdot \mathbf{r}_{n},
$$


from which the (dynamically irrelevant) penalty term has disappeared. Introducing now the matrix $\widetilde{\mathbf{A}}=\left(\widetilde{A}_{i j}\right)$ through

$$
\widetilde{\mathbf{A}}=\mathbf{G W G}^{T},
$$

and inserting Eq. (26) into Eq. (21) gives

$$
\zeta \frac{\partial}{\partial t} y_{j}(t)+K \sum_{k=1}^{N} \widetilde{A}_{j k} y_{k}(t)=f_{j}(t) .
$$

The solution of the set of Eq. (28) requires the diagonalization of the matrix $\widetilde{\mathbf{A}}$, which can be performed analytically: Based on Eqs. (6) and (23) it is a simple matter to check that $\mathbf{W}$ commutes both with $\mathbf{G}$ and with $\mathbf{G}^{T}$. (One can also show that the matrices $\mathbf{G}$ and $\mathbf{G}^{T}$ can be diagonalized using $\mathbf{C}$ of Eq. (13), leading to the eigenvalues $[\exp (-\mathrm{i} 2 \pi k / N)-1]$ and $[\exp (\mathrm{i} 2 \pi k / N)-1]$ where $k=1, \ldots, N$, respectively.)

Hence, introducing the (Rouse) Laplacian matrix $\mathbf{A} \equiv \mathbf{G G}^{T}$ for the flexible ring, ${ }^{51-54}$

$$
\mathbf{A}=\left(\begin{array}{cccccc}
2 & -1 & 0 & \ldots & 0 & -1 \\
-1 & 2 & -1 & 0 & \ldots & 0 \\
0 & \ddots & \ddots & \ddots & \ddots & \vdots \\
\vdots & \ddots & \ddots & \ddots & \ddots & 0 \\
0 & \ldots & 0 & -1 & 2 & -1 \\
-1 & 0 & \ldots & 0 & -1 & 2
\end{array}\right),
$$

one can remark, based on Eq. (23), that because of the form of $\mathbf{G}$ and since $\mathbf{W}$ is a periodically symmetric matrix, see Eq. (6), $\mathbf{G}^{T}$ and $\mathbf{W}$ commute, i.e., $\mathbf{W G}^{T}=\mathbf{G}^{T} \mathbf{W}$. Thus, from Eq. (27) follows

$$
\widetilde{\mathbf{A}}=\mathbf{A W}
$$

Moreover, the matrix $\mathbf{W}$ can also be written in terms of $\mathbf{A}$. As can be readily verified by comparing Eqs. (6) and (29), one has

$$
\mathbf{W}=(\alpha+2 \beta+2 \gamma) \mathbf{1}-(\beta+4 \gamma) \mathbf{A}+\gamma \mathbf{A}^{2} .
$$

Together with Eq. (30) this leads to

$$
\widetilde{\mathbf{A}}=(\alpha+2 \beta+2 \gamma) \mathbf{A}-(\beta+4 \gamma) \mathbf{A}^{2}+\gamma \mathbf{A}^{3} .
$$

Thus, the matrices $\mathbf{W}, \mathbf{A}$, and $\widetilde{\mathbf{A}}$ share the same eigenvectors, which are given by Eq. (13). Indeed, comparing Eq. (29) with Eq. (6), one immediately realizes that $\mathbf{A}$ is a particular case of $\mathbf{W}$ with $\alpha=2, \beta=-1$, and $\gamma=0$. Hence, from Eq. (14) follow the well-known ${ }^{33,51-54}$ eigenvalues $\left\{\xi_{k}\right\}$ of $\mathbf{A}$,

$$
\xi_{k}=4 \sin ^{2}\left(\frac{\pi k}{N}\right)
$$

most of which are doubly degenerate, given that $\xi_{k}=\xi_{N-k}$. Based on Eqs. (30) and (32), the full set of eigenvalues of $\widetilde{\mathbf{A}}$ reads then

$$
\begin{aligned}
\varphi_{k} & =\xi_{k} \lambda_{k}=(\alpha+2 \beta+2 \gamma) \xi_{k}-(\beta+4 \gamma) \xi_{k}^{2}+\gamma \xi_{k}^{3}, \\
\text { for } k & =1, \ldots, N .
\end{aligned}
$$

Given that both $\xi_{k}$ and $\lambda_{k}$ are positive for $k=1, \ldots,(N-1)$ and that only the eigenvalue $\varphi_{N}$ vanishes, $\varphi_{N}=\xi_{N} \lambda_{N}=0$, the matrix $\widetilde{\mathbf{A}}$ is non-negative definite.
We remark that in the limit of $\gamma \rightarrow 0$ we obtain for $\widetilde{\mathbf{A}}$ and for $\varphi_{k}$ the known results for semiflexible rings without $\mathrm{DR}^{33}$ (in line with the formalism of Bixon and Zwanzig ${ }^{1}$ ); the limit of both $\gamma \rightarrow 0$ and $\beta \rightarrow 0$ leads to flexible rings, ${ }^{51,52}$ in the sense of the Rouse model, ${ }^{48}$ see Appendix $\mathrm{C}$ for details.

Furthermore, we note that the Laplacian $\mathbf{A}=\left(A_{i j}\right)$ is a discrete version of a second derivative, ${ }^{48} \sum_{k} A_{n k} y_{k} \rightarrow \frac{\partial^{2} y_{n}}{\partial n^{2}}$. Thus, the matrix $\widetilde{\mathbf{A}}$ is related to a linear combination of second, fourth, and sixth order differential operators, whose Fourier transforms are the second, the fourth, and the sixth powers of the wave number, respectively. A continuum picture emerges in the limit of $N$ large, $N \gg 1$. In this limit, by expanding $\xi_{k}$ of Eq. (33) up to sixth order in $k$ and inserting it into Eq. (34) yields

$$
\varphi_{k} \approx A_{1}\left(\frac{2 \pi k}{N}\right)^{2}+A_{2}\left(\frac{2 \pi k}{N}\right)^{4}+A_{3}\left(\frac{2 \pi k}{N}\right)^{6},
$$

where

$$
\begin{gathered}
A_{1}=\alpha+2 \beta+2 \gamma, \\
A_{2}=-(\alpha+14 \beta+50 \gamma) / 12,
\end{gathered}
$$

and

$$
A_{3}=(\alpha+62 \beta+602 \gamma) / 360 .
$$

We note that Eq. (35) is valid for $k \ll N / 2$ and that the coefficients $\left\{A_{i}\right\}$ are uniquely determined through the parameters $(\alpha, \beta, \gamma)$. The regions of $k$ where $\varphi_{k} \sim k^{s}(s=2,4,6)$ are discussed in Appendix B, see Eqs. (B1)-(B3).

\section{Mean-square displacement}

We turn now to the determination of the MSD $\Psi_{n}(t)$ of the ring monomers. The MSD, say, of the $n$th monomer $(n=1 \ldots N)$, is given through ${ }^{48}$

$$
\Psi_{n}(t) \equiv\left\langle\left[\mathbf{r}_{n}(t)-\mathbf{r}_{n}(0)\right]^{2}\right\rangle .
$$

The MSD is calculated then by making use the normal mode expansion $^{48}$

$$
\mathbf{r}_{n}(t)=\sum_{j=1}^{N} C_{n j} \mathbf{Q}_{j}(t) .
$$

Here, the elements $C_{n j}$ of the matrix $\mathbf{C}$ are given by Eq. (13) and for them $\left|C_{n j}\right|^{2}=1 / N$ holds. Moreover, we recall that $\mathbf{C}$ diagonalizes $\widetilde{\mathbf{A}}, \mathbf{C}^{-1} \widetilde{\mathbf{A}} \mathbf{C}=\operatorname{Diag}\left(\varphi_{1} \ldots \varphi_{N}\right)$. Thus, under the transformation given by Eq. (40) the Langevin equation simplifies to

$$
\zeta \frac{\partial}{\partial t} Q_{\alpha k}(t)+K \varphi_{k} Q_{\alpha k}(t)=\hat{f}_{\alpha k}(t),
$$

where $\alpha$ is the spatial coordinate, the $\left\{\varphi_{k}\right\}$ are the eigenvalues of $\widetilde{\mathbf{A}}$, Eq. (34), and $\hat{f}_{\alpha k}(t)=\sum_{i}\left(\mathbf{C}^{-1}\right)_{k i} f_{\alpha i}(t)$. Hence, $\left\langle\hat{f}_{\alpha k}(t) \hat{f}_{\beta m}\left(t^{\prime}\right)\right\rangle=2 k_{B} T \zeta \delta_{k m} \delta_{\alpha \beta} \delta\left(t-t^{\prime}\right)$ and $\left\langle\hat{\mathbf{f}}_{k}(t)\right\rangle=0$, so that, from Eq. (41) the following relations hold: ${ }^{24,48}$

$$
\left\langle\left(Q_{\alpha N}(t)-Q_{\alpha N}(0)\right)\left(Q_{\beta N}(t)-Q_{\beta N}(0)\right)\right\rangle=\delta_{\alpha \beta} \frac{2 k_{B} T}{N \zeta} t
$$


and

$$
\left\langle Q_{\alpha k}(t) Q_{\beta m}(0)\right\rangle=\delta_{\alpha \beta} \delta_{k m}\left\langle Q_{\beta m}^{2}\right\rangle e^{-t / \tau_{m}}(k, m=1, \ldots, N-1),
$$

where $\left\langle Q_{\beta m}^{2}\right\rangle=k_{B} T /\left(K \varphi_{m}\right)$ and $\tau_{m}=\zeta /\left(K \varphi_{m}\right)=\tau_{0} / \varphi_{m}$. Inserting Eq. (40) into (39) and using Eqs. (42) and (43), we obtain

$$
\Psi(t) \equiv \Psi_{n}(t)=\frac{2 l^{2}}{N \tau_{0}} t+\frac{2 l^{2}}{N} \sum_{k=1}^{N-1} \frac{1-\exp \left(-\varphi_{k} t / \tau_{0}\right)}{\varphi_{k}} .
$$

Thus, the MSD for homogeneous rings is independent on $n$. Moreover, it depends only on the eigenvalues $\left\{\varphi_{k}\right\}$, Eq. (34), but not on the eigenvectors of $\widetilde{\mathbf{A}}$.

From Eqs. (B6) to (B10) of Appendix B $\Psi(t)$ shows several distinct behaviors: At very short times, $t \ll \min \left(\tau_{0} / \varphi_{k}\right)$, the monomers perform a diffusive motion, unhindered by their neighbors, $\Psi(t) \simeq 2 l^{2}\left(t / \tau_{0}\right)$. Next in line, the monomers start to feel the dihedral restrictions, which are related to hindered rotations, leading then to $\Psi(t) \simeq\left(2 l^{2} / 5 \pi\right) \Gamma(1 / 6) A_{3}^{-1 / 6}\left(t / \tau_{0}\right)^{5 / 6}$, with $A_{3}$ as in Eq. (38). Then the $\Psi(t)$ enters the bending regime, $\Psi(t)$ $\simeq\left(2 l^{2} / 3 \pi\right) \Gamma(1 / 4) A_{2}^{-1 / 4}\left(t / \tau_{0}\right)^{3 / 4}$, with $A_{2}$ as in Eq. (37). We note that the above mentioned regimes involve the local scales, evident since the $\Psi(t)$-forms do not depend on $N$. The last subdiffusive regime (known from the Rouse model ${ }^{24,48}$ ) always exists for very long rings and disappears for short, stiff ones. Here, the MSD follows $\Psi(t) \simeq\left(2 l^{2} / \sqrt{\pi}\right) A_{1}^{-1 / 2}\left(t / \tau_{0}\right)^{1 / 2}$ as in $A_{1}$ from Eq. (36). Finally, at very long times, $\left(t / \tau_{0}\right)$ $\gg N^{2}$, the monomers follow the global motion of their ring and $\Psi(t) \simeq\left(2 l^{2} / N\right)\left(t / \tau_{0}\right)$, which is again a diffusive regime. The time boundaries, in which the subdiffusive regimes $\Psi(t)$ $\sim t^{\mu}(\mu<1)$ are valid, depend on the values of the coefficients $\left\{A_{i}\right\}$, see Appendix $\mathrm{B}$. While the subdiffusive ranges with $\mu=1 / 2$ and $3 / 4$ are well-known from the literature for flexible and for semiflexible chains, ${ }^{6,13,48}$ the $\mu=5 / 6$ range is a hallmark of the dihedral restrictions.

\section{NONHOMOGENEOUS RINGS}

In this section, we extend the model of Secs. II and III to nonhomogeneous semiflexible rings. Such rings arise naturally when considering a semiflexible chain whose endpoints coalesce without putting any restriction on their neighboring bonds. The ensuing ring has then a site at which the orientational restrictions do not hold, breaking thus the cyclical symmetry.

The starting point is again the linear chain ${ }^{28}$ discussed in Sec. II. Closing such a chain into a ring and following the procedure of Sec. II, the bonds $\left\{\mathbf{d}_{a}\right\}$ of the nonhomogeneous ring obey now (in spirit of Eq. (10)) the distribution

$$
\widetilde{P}\left(\left\{\mathbf{d}_{a}\right\}\right)=\frac{1}{\widetilde{Z}} \exp \left(-\frac{3}{2 l^{2}} \sum_{a, b} \widehat{\widetilde{W}}_{a b} \mathbf{d}_{a} \cdot \mathbf{d}_{b}\right) .
$$

Since no new conditions are imposed on the bonds neighboring the closure site, the matrix $\widehat{\widetilde{\mathbf{W}}}$ in Eq. (45) reads

$$
\widehat{\widetilde{\mathbf{W}}}=\widetilde{\mathbf{W}}+\frac{1}{\epsilon^{2}} \mathbf{L}
$$

where $\tilde{\mathbf{W}}$ is given by Eq. (5) and $\mathbf{L}$ and $\epsilon$ are as in Eq. (11). Given that we work with a penalty function, the bonds $\left\{\mathbf{d}_{a}\right\}$ represent still an independent set of variables. So that using the distribution $\widetilde{P}$ of Eq. (45) while keeping $\epsilon>0$, the average values of $\mathbf{d}_{a} \cdot \mathbf{d}_{b}$ are given through

$$
\left\langle\mathbf{d}_{a} \cdot \mathbf{d}_{b}\right\rangle=l^{2}\left(\widehat{\widetilde{\mathbf{W}}}^{-1}\right)_{a b} .
$$

In Appendix B, we show that in the limit $\epsilon \rightarrow 0^{+}$one has

$$
\left(\widehat{\widetilde{\mathbf{W}}}^{-1}\right)_{a b}=\left(\tilde{\mathbf{W}}^{-1}\right)_{a b}-\frac{\sum_{k}\left(\tilde{\mathbf{W}}^{-1}\right)_{a k} \sum_{n}\left(\tilde{\mathbf{W}}^{-1}\right)_{n b}}{\sum_{n, k}\left(\widetilde{\mathbf{W}}^{-1}\right)_{n k}} .
$$

We note that Eq. (48) can also be obtained by considering general Gaussian distributions under strict (holonomic) constraints, ${ }^{36}$ fact recently used in the treatment of nonMarkovian diffusion-limited reactions. ${ }^{37-39}$ Given that the $\left\{\left(\widetilde{\mathbf{W}}^{-1}\right)_{a b}\right\}$ are known in closed form, ${ }^{28}$ the conditions on the local bond orientations represented through $\left\langle\mathbf{d}_{a} \cdot \mathbf{d}_{b}\right\rangle$ can be expressed as function of the parameters $\widetilde{q}_{1}$ and $\widetilde{q}_{2}$ of the chain, see Appendix B. Here, we remark, however, that for nonhomogeneous rings, in contrast to Eqs. (18)-(20), the circular symmetry does not hold anymore, $\left\langle\mathbf{d}_{a+k} \cdot \mathbf{d}_{b+k}\right\rangle \neq\left\langle\mathbf{d}_{a} \cdot \mathbf{d}_{b}\right\rangle$.

The procedure for analyzing the dynamics of the nonhomogeneous ring follows now closely the procedure for the homogeneous ring described in Sec. III B. Thus, we obtain the dynamical matrix $\widehat{\mathbf{A}}$ of the nonhomogeneous ring using the transformation Eq. (22) and performing the steps of Eqs. (24)-(26). The resulting dynamical matrix again takes the form

$$
\widehat{\mathbf{A}}=\mathbf{G} \widetilde{\mathbf{W}} \mathbf{G}^{T},
$$

where $\mathbf{G}$ is given by Eq. (23). Similar to Eq. (32), the matrix $\widehat{\mathbf{A}}$ can be represented through the matrix $\mathbf{A}$ of the flexible ring, Eq. (29), but with an additional matrix term which accounts for the ring's inhomogeneity. Namely, one has

$$
\widehat{\mathbf{A}}=\widetilde{\mathbf{A}}+\boldsymbol{\Delta}
$$

where the matrix $\widetilde{\mathbf{A}}$ has the functional form given by Eq. (32) and the matrix $\boldsymbol{\Delta}=\left(\Delta_{i j}\right)$ has the following elements:

$$
\Delta_{11}=2\left(\alpha^{\prime}-\alpha+\beta\right) \text {, }
$$

$$
\begin{gathered}
\Delta_{12}=\Delta_{21}=\Delta_{1 N}=\Delta_{N 1}=\beta^{\prime}-2 \beta+\gamma-\alpha^{\prime}+\alpha, \\
\Delta_{13}=\Delta_{31}=\Delta_{1, N-1}=\Delta_{N-1,1}=\beta-\beta^{\prime}-\gamma, \\
\Delta_{22}=\Delta_{N N}=2 \beta-2 \beta^{\prime}+\alpha+\alpha^{\prime \prime}-2 \alpha, \\
\Delta_{23}=\Delta_{32}=\Delta_{N, N-1}=\Delta_{N-1, N}=\beta^{\prime}-\beta-\alpha^{\prime \prime}+\alpha, \\
\Delta_{2, N-1}=\Delta_{N-1,2}=\Delta_{N, 3}=\Delta_{3, N}=\gamma, \\
\Delta_{2, N}=\Delta_{N, 2}=\beta-2 \gamma, \\
\Delta_{33}=\Delta_{N-1, N-1}=\alpha^{\prime \prime}-\alpha .
\end{gathered}
$$


All other elements of $\Delta$ vanish. The parameters of Eqs. (51)(58) as function of $\widetilde{q}_{1}$ and $\widetilde{q}_{2}$ are given in Eqs. (A1)-(A6) in Appendix A.

Also as in Sec. III C, we focus here on the MSD of ring monomers. Proceeding similarly to Eqs. (39)-(44), we obtain the MSD of the $n$th monomer of the nonhomogeneous ring,

$$
\widehat{\Psi}_{n}(t)=\frac{2 l^{2}}{N \tau_{0}} t+2 l^{2} \sum_{k=1}^{N-1} \widehat{C}_{n k}^{2} \frac{1-\exp \left(-\widehat{\varphi}_{k} t / \tau_{0}\right)}{\widehat{\varphi}_{k}} .
$$

In Eq. (59), the $\widehat{C}_{n k}$ are the elements of a matrix $\widehat{\mathbf{C}}=\left(\widehat{C}_{n k}\right)$ which diagonalizes $\widehat{\mathbf{A}}$. We denote the eigenvalues of $\widehat{\mathbf{A}}$ by $\left\{\widehat{\varphi}_{k}\right\}$, with $\widehat{\varphi}_{N}=0$. The eigenvector corresponding to $\widehat{\varphi}_{N}$ is $(1 \ldots 1) / \sqrt{N}$, as can be easily checked using Eqs. (50)-(58). Here we remark, however, that in contrast to Eq. (44), in the nonhomogeneous case the MSD depends on the monomer's position in the ring. Therefore, we introduce the structural average of MSD as ${ }^{55,56}$

$$
\langle\widehat{\Psi}(t)\rangle \equiv \frac{1}{N} \sum_{n=1}^{N} \widehat{\Psi}_{n}(t) .
$$

Using that the eigenvectors which build up the matrix $\widehat{\mathbf{C}}$ are orthonormal, i.e., $\sum_{n=1}^{N} \widehat{C}_{n k}^{2}=1$, we obtain (due to the fluctuation-dissipation theorem, akin to Refs. 55 and 56)

$$
\langle\widehat{\Psi}(t)\rangle=\frac{2 l^{2}}{N \tau_{0}} t+\frac{2 l^{2}}{N} \sum_{k=1}^{N-1} \frac{1-\exp \left(-\widehat{\varphi}_{k} t / \tau_{0}\right)}{\widehat{\varphi}_{k}} .
$$

Equation (61) has the same functional form as Eq. (44), the difference residing in the eigenvalues $\left\{\widehat{\varphi}_{k}\right\}$. The difference be-
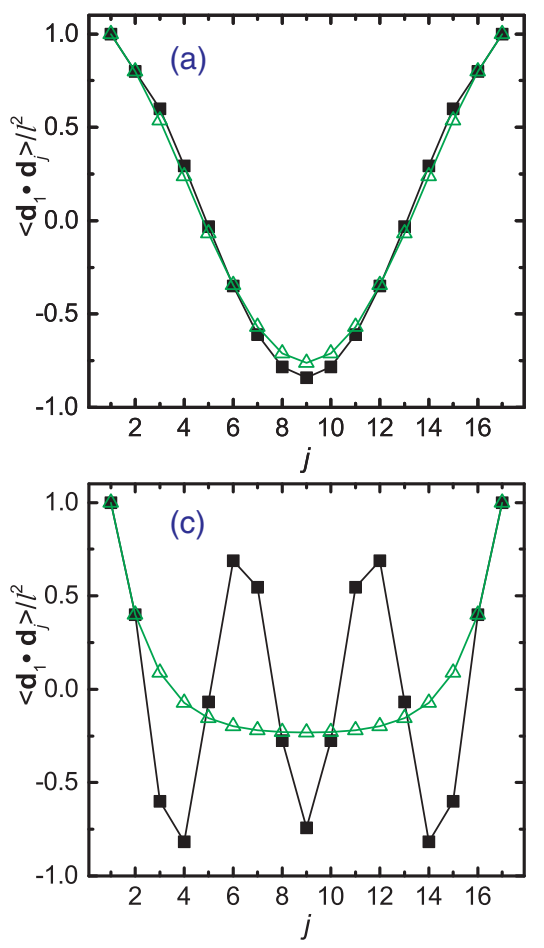

tween $\langle\widehat{\Psi}(t)\rangle$, Eq. (61), of nonhomogeneous rings and $\Psi(t)$, Eq. (44), of homogeneous ones is discussed in Sec. V.

\section{RESULTS AND DISCUSSION}

In this section, we discuss the statics and dynamics of the rings modeled in Secs. III and IV. First, we focus on the homogeneous rings and discuss the role of dihedral restrictions. Then we compare nonhomogeneous rings with homogeneous ones.

\section{A. Influence of dihedral restrictions on statics and on dynamics of homogeneous semiflexible rings}

In this subsection, we study the role of DR by comparing the static and dynamical behaviors of homogeneous rings with DR of Sec. III with those of rings without DR, i.e., rings with freely rotating bonds. The latter model is a traditional approach to semiflexible rings, ${ }^{5,33,57}$ in which the semiflexibility is modeled through a single parameter $q_{1}$ related to the angular restrictions. Thus, the traditional model is a particular case of Sec. III in which the constraints of Eq. (20) are relaxed by setting $\gamma=0$, see Appendix C.

We start by investigating of the local properties of homogeneous rings, by considering their equilibrium bond-bond orientations $\left\langle\mathbf{d}_{1} \cdot \mathbf{d}_{j}\right\rangle$, calculated based on Eq. (17) for rings with DR and on Eq. (C1) for those without DR. Here, we take the number of bonds (or beads) to be $N=16$.

In parts (a)-(d) of Fig. 3, we plot in black the normalized bond-bond correlations of rings with DR for different stiffness parameter sets $\left(q_{1}, q_{2}\right)$ : $(0.8,0.6),(0.6,-0.2)$,
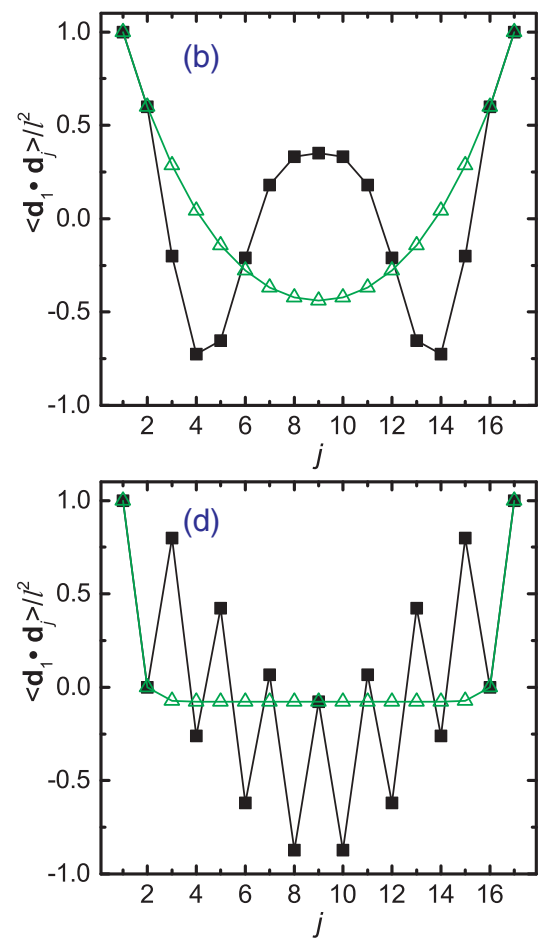

FIG. 3. Bond-bond correlations of homogeneous rings composed of $N=16$ segments under DR (black squares), for different sets of stiffness parameters $\left(q_{1}, q_{2}\right):(0.8,0.6)(\mathrm{a}),(0.6,-0.2)(\mathrm{b}),(0.4,-0.6)(\mathrm{c})$, and $(0,0.8)(\mathrm{d})$. The results are compared with those for rings without DR for the same $q_{1}$ (green triangles), see text for details. The lines are guides for the eye. 
(a)

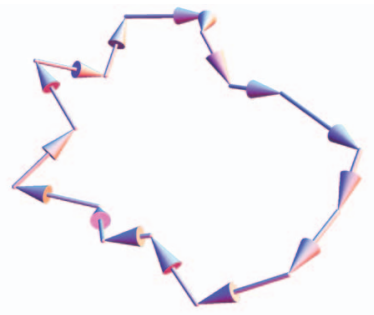

(c)

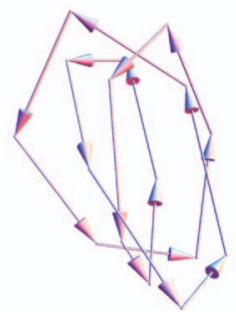

(b)

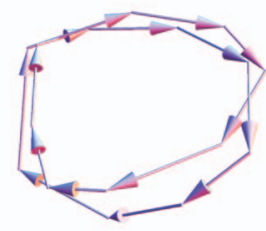

(d)

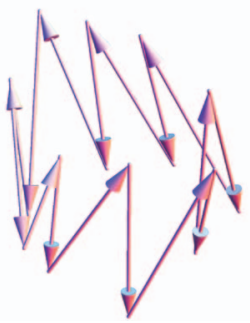

FIG. 4. Configurations of $N=16$ bonds of homogeneous rings under DR, corresponding to the different stiffness parameters sets (a)-(d) of Fig. 3.

$(0.4,-0.6)$, and $(0,0.8)$. For rings without DR the values of the single stiffness parameter $q_{1}$ are chosen to be the same as the $q_{1}$ for the rings with DR and their correlation functions are displayed in green in Fig. 3. Now, Fig. 3 evidences the symmetry of all curves around the site $j=9$. However, while the correlation functions of rings with DR display in parts (a)(d) one, two, three, and eight local minima, respectively, for rings without DR the curves have always only one minimum. The most significant difference between the orientational correlation functions is given in part (d) of Fig. 3, where for the rings with DR it shows strong oscillations, whereas for rings without DR it practically disappears at long distances.

In order to have a closer look at the results of Fig. 3, we display in Fig. 4 randomly chosen conformations of rings with DR taken from the bonds' distributions calculated for stiffness parameter sets $\left(q_{1}, q_{2}\right)$ which are the same as in parts (a)(d) of Fig. 3, respectively. Interestingly, in part (d) the bonds look like a zigzag chain embedded in a cylindrical surface. (In fact, the bond-bond orientation function of a fully symmetric zigzag ring is close to that of Fig. 3(d).) Thus, by fixing the orientations between the NN and the NNN bonds, we may influence the distances between sites and hence render the structures more or less compact. In particular, by letting $q_{2}$ be negative, we create an incentive for antiparallel orientations of the NNN bonds. Whether and to which extent this happens depends on a subtle interplay between the $q_{1}$ and the $q_{2}$ parameters, in particular the tendency to an antiparallel orientation of NNN bonds is stronger when $q_{2}$ gets close to the value -1 . What is evident, however, is that such a tendency leads to an increase of the local density so that the rings get more compact, fact reflected in their radii of gyration as we proceed to show.

In Table I, we list the $\left\langle R_{g}^{2}\right\rangle / l^{2}$ of rings with and without DR, calculated using Eq. (B13) of Appendix B 3 based on the $\left\{\left\langle\mathbf{d}_{a} \cdot \mathbf{d}_{b}\right\rangle\right\}$ of Fig. 3. In case A, the gyration radii for rings with and without DR are close to each other (as also holds for Fig. 3), whereas in all other cases they differ significantly. Rings without DR are controlled through the single parameter

TABLE I. Gyration radii of semiflexible rings with $N=16$ for the models (a)-(d) of Fig. 3 and of the fully flexible $N=16$ ring. For the latter $q_{1}=q_{2}$ $=-1 / 15$, see Refs. 33 and 59 and also the text for details.

\begin{tabular}{lcccc}
\hline \hline & A & B & C & D \\
\hline Rings with DR & 5.829 & 1.549 & 1.035 & 3.432 \\
Rings without DR & 5.578 & 4.132 & 2.978 & 1.570 \\
Fully-flexible ring & \multicolumn{4}{c}{1.417} \\
\hline \hline
\end{tabular}

$q_{1}$, related to the NN bond interactions; their $\left\langle R_{g}^{2}\right\rangle$ decreases monotonically when $q_{1}$ gets smaller (i.e., with increasing flexibility) and has as limiting value $\left\langle R_{g}^{2}\right\rangle / l^{2}=(N+1) / 12$ $\approx 1.417$, which holds for fully flexible rings. ${ }^{58,59}$ Rings with DR include two types of restrictions (for the NN and for the NNN bonds), which are controlled through the two parameters $q_{1}$ and $q_{2}$. This leads to a very rich picture: For example, the choice $q_{1}=0$ and $q_{2}=0.8$ (case D) does not lead to a flexible situation, but to a somewhat parallel orientation of the NNN bonds. Also, the smaller $\left\langle R_{g}^{2}\right\rangle$ in the cases B and C is not a sign that the rings are more flexible; in fact, their small $\left\langle R_{g}^{2}\right\rangle$ is due to the tendency to an antiparallel orientation of the NNN bonds, which renders the rings more compact. Exemplarily, in case $\mathrm{C}$, the $\left\langle R_{g}^{2}\right\rangle$ of the semiflexible ring with DR is even smaller than that of the fully flexible ring of the same length $N$.

We turn now to the dynamics of rings, by considering the MSD $\Psi(t)$ of their monomers, see Eq. (44). In Fig. 5, we plot the $\Psi(t)$ for $N=1000$ rings with and without DR. The stiffness parameters $q_{1}=0.72$ and $q_{2}=0.14$ are chosen in such a way that for the ring with DR (black line) one gets a clear scaling of $t^{5 / 6}$. The related MSD of the ring without DR with stiffness parameter $q_{1}=0.72$ (green line) shows in this time domain a $t^{3 / 4}$-behavior. We note that the dynamical matrix of rings with DR contains (due to the NNN bond interactions) a term proportional to the third power of the Laplacian matrix, see Eq. (32). This term leads to the $\Psi(t) \sim t^{5 / 6}$ behavior, see Appendix B 2 for details. In the absence of DR, the parameter $\gamma$ in Eq. (32) vanishes and this behavior disappears. Both rings, being quite long, show a $t^{1 / 2}$-behavior, but with

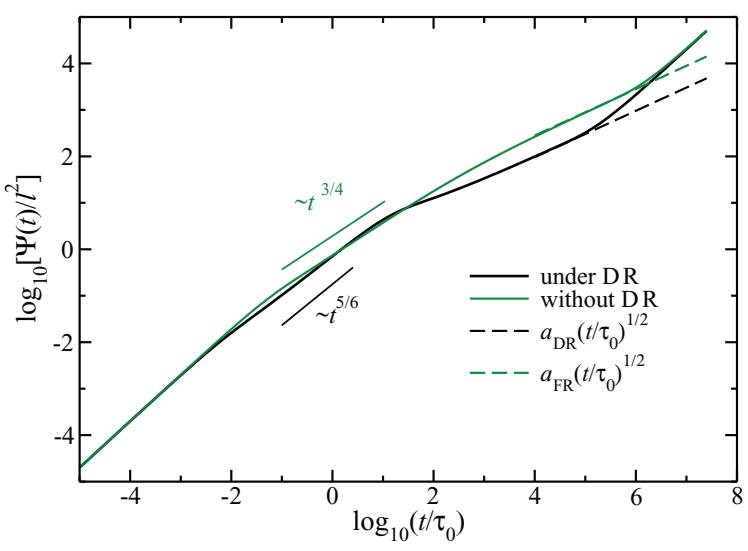

FIG. 5. Mean-square monomer displacement $\Psi(t)$ for a homogeneous ring under DR (black solid line) and for a ring without DR (green solid line). In both cases, the rings have $N=1000$ bonds, $q_{1}=0.72$ and for the ring under DR $q_{2}=0.14$. Note that the dashed lines have the slope $t^{1 / 2}$, see text for details. 
significantly distinct subdiffusive coefficients $\left(a_{D R} \approx 0.956\right.$ for the ring under DR and $a_{F R} \approx 2.814$ for that without DR, see Fig. 5).

We conclude that the DR influence very much the statics and the dynamics of rings, which show, as a function of the $\mathrm{DR}$, essential differences in their qualitative and quantitative behaviors.

\section{B. Statics and dynamics of nonhomogeneous rings}

In this subsection, we consider the static and the dynamical properties of nonhomogeneous rings with DR, which were discussed in Sec. IV.

As in Sec. V A, the static properties of nonhomogeneous rings are studied through the $\left\langle\mathbf{d}_{1} \cdot \mathbf{d}_{j}\right\rangle$ calculated based on Eqs. (47) and (48). Fig. 6 presents the normalized bondbond correlations of nonhomogeneous rings consisting of $N=16$ segments (color-coded by red) compared to those of the homogeneous rings displayed on parts (a)-(d) of Fig. 3 (black). We consider nonhomogeneous rings which have $\left\langle\mathbf{d}_{1} \cdot \mathbf{d}_{2}\right\rangle / \sqrt{\left\langle\mathbf{d}_{1}^{2}\right\rangle\left\langle\mathbf{d}_{2}^{2}\right\rangle}=q_{1}$ and $\left\langle\mathbf{d}_{1} \cdot \mathbf{d}_{3}\right\rangle / \sqrt{\left\langle\mathbf{d}_{1}^{2}\right\rangle\left\langle\mathbf{d}_{3}^{2}\right\rangle}$ $=q_{2},\left(q_{1}, q_{2}\right)$ are as in Fig. 3. Now, Fig. 6 evidences for the nonhomogeneous rings a loss of symmetry of the correlation functions observed for homogeneous rings with respect to the site $j=9$. The nonhomogeneous rings first follow qualitatively the behavior of the homogeneous rings, however, the absence of direct restrictions between the terminal bonds of nonhomogeneous rings leads to the damping of the correlations along the ring. As a result, the positions of the local minima and maxima on parts (a)-(c) are shifted, the correlations between the $\mathbf{d}_{1}$ and $\mathbf{d}_{N}$ of the nonhomogeneous rings being significantly smaller (especially in cases (a) and (d)) than those of the homogeneous ones.

Now, we turn to the dynamics of nonhomogeneous rings. In Fig. 7, we compare the MSD $\Psi(t)$ of homogeneous rings,
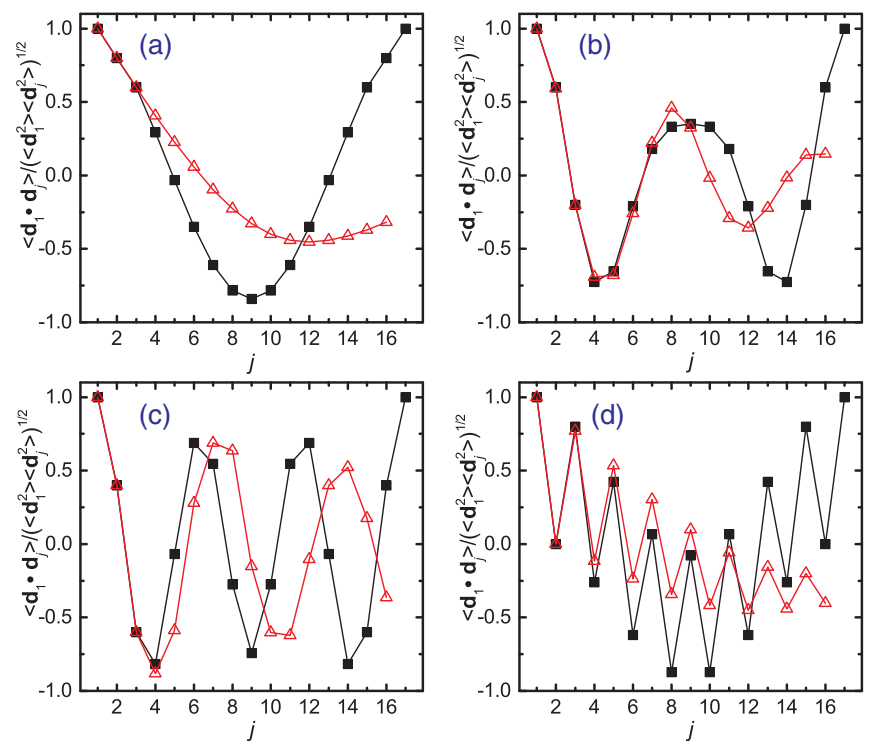

FIG. 6. Bond-bond correlations in homogeneous rings (black squares) and in nonhomogeneous rings (red open triangles) consisting of $N=16$ segments. The cases (a)-(d) are as in of Fig. 3 and the lines are guides for the eye, see text for details.
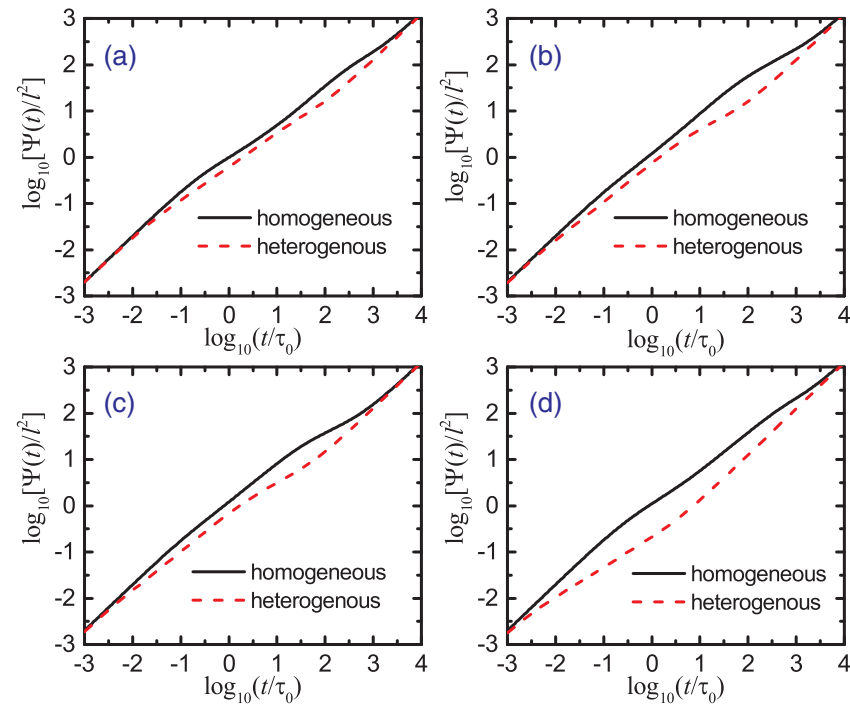

FIG. 7. Mean-square displacements $\Psi(t)$ of monomers of homogeneous rings (black solid lines) and $\langle\widehat{\Psi}(t)\rangle$ of nonhomogeneous rings (red dashed lines). The rings consist of $N=16$ segments and the cases (a)-(d) are as in Fig. 6, see text for details.

Eq. (44), with structural average of MSD $\langle\widehat{\Psi}(t)\rangle$ of nonhomogeneous ones, Eq. (61). We note that structural averaging of $\Psi(t)$ of homogeneous rings keeps Eq. (44) unchanged. The parameters in parts (a)-(d) are chosen the same as in parts (a)(d) of Fig. 6. As can be inferred from the figure, for very short and for very long times the plots for the rings coincide, showing that these time regions do not depend on the eigenvalue spectra. The particular properties of rings appear in the intermediate time domain. ${ }^{24}$ Here, we see for all parts of Fig. 7 clear differences between the plots, the largest difference being in part (d). We note that for part (d), as discussed in Sec. V A, the dihedral restrictions are most important. Comparing parts (b) and (c) one can observe that the difference between the curves shifts from longer to shorter times. This property is related to the fact that parts (b) and (c) are related to more compact rings, which reach faster the diffusive regime, where no discrepancy between the homogeneous and nonhomogeneous rings is noticeable.

We conclude that the presence of a single inhomogeneity significantly changes the static and dynamic properties of rings, especially in cases with strong dihedral restrictions.

\section{CONCLUSIONS}

In this article, we studied semiflexible ring polymers by focusing on their creation from linear polymers through cyclization. For this we imposed closure constraints based on a penalty potential, a method which is quite general and which allows to study arbitrary Gaussian rings under different conditions. Here, we used the semiflexibility model of Ref. 28 which takes both angular and dihedral restrictions into account. As we have shown, the dihedral restrictions have a clear influence on the static and dynamical properties of rings.

Using the circular symmetry of homogeneous rings, we obtained analytic results for their dynamical matrix and for its eigenvalue spectrum. Based on these results we studied the behavior of the mean-square displacement $\Psi(t)$ of the rings' 
monomers in different time domains. Thus, we could observe in the intermediate time regime three different subdiffusive behaviors, going as $\Psi(t) \sim t^{\mu}$ with $\mu=1 / 2,3 / 4$, and 5/6. The well-known 1/2-regime is related to fully flexible chains, ${ }^{48}$ the exponent $3 / 4$ is the hallmark of semiflexible chains with angular (bending) interactions, ${ }^{6,13}$ while the dihedral restrictions lead to the exponent $5 / 6$.

Our closure method is very general and it allows, in particular, to also study nonhomogeneous rings. Here, we treated a situation where the symmetry was broken at a single site. As we showed, such an asymmetry shows up both in the static and in the dynamical features of nonhomogeneous rings, especially when the DR are large. Concluding, we are led to the conviction that the method which we employed in this work could open new pathways for the theoretical study of arbitrary semiflexible polymers with loops.

\section{ACKNOWLEDGMENTS}

M.D. and A.B. acknowledge the support of the Deutsche Forschungsgemeinschaft (DFG) through Grant No. B1 142/11-1, of the DAAD through the PROCOPE program (Project No. 55853833), of the Marie Curie International Research Staff Exchange Scheme Fellowship within the 7th European Community Framework Program SPIDER (PIRSES-GA-2011-295302), as well as additional funding from the Fonds der Chemischen Industrie. T.G., O.B., and R.V. acknowledge the support of Campus France (Project No. 28252XE) and of the European Research Council starting Grant No. FPTOpt-277998.

\section{APPENDIX A: MATRIX W WF A LINEAR CHAIN}

We recall here that for a chain the entries of $\widetilde{\mathbf{W}}$, Eq. (5) of the main text, are known in closed form as a function of stiffness parameters $\widetilde{q}_{1}$ and $\widetilde{q}_{2} \cdot{ }^{28}$ Setting $\tilde{p}=\left(\widetilde{q}_{2}-\widetilde{q}_{1}^{2}\right)$ $/\left(1-\widetilde{q}_{1}^{2}\right)$ the diagonal elements of $\widetilde{\mathbf{W}}$ are $^{28}$

$$
\begin{aligned}
\alpha^{\prime} & =\frac{1}{\left(1-\widetilde{q}_{1}^{2}\right)\left(1-\widetilde{p}^{2}\right)}, \\
\alpha^{\prime \prime} & =\frac{1+(1-\widetilde{p})^{2} \widetilde{q}_{1}^{2}}{\left(1-\widetilde{q}_{1}^{2}\right)\left(1-\widetilde{p}^{2}\right)},
\end{aligned}
$$

and

$$
\alpha=\frac{1+\widetilde{p}^{2}+(1-\tilde{p})^{2} \widetilde{q}_{1}^{2}}{\left(1-\widetilde{q}_{1}^{2}\right)\left(1-\widetilde{p}^{2}\right)} .
$$

Furthermore, the elements of $\tilde{\mathbf{W}}$ related to nearestneighboring bonds are $\mathrm{a}^{28}$

$$
\beta^{\prime}=-\frac{\widetilde{q}_{1}}{\left(1-\widetilde{q}_{1}^{2}\right)(1+\widetilde{p})}
$$

and

$$
\beta=-\frac{\widetilde{q}_{1}(1-\tilde{p})}{\left(1-\widetilde{q}_{1}^{2}\right)(1+\widetilde{p})}
$$

Moreover, the elements of $\tilde{\mathbf{W}}$ related to next-nearestneighboring bonds are ${ }^{28}$

$$
\gamma=-\frac{\tilde{p}}{\left(1-\widetilde{q}_{1}^{2}\right)\left(1-\widetilde{p}^{2}\right)}
$$

\section{APPENDIX B: CALCULATION DETAILS}

\section{Eigenvalue spectra of homogeneous rings in the limit of large $N$}

The eigenvalues of homogeneous rings are given by the $\left\{\varphi_{k}\right\}$ of Eq. (34). Here, we analyze their behavior at large $N$, i.e., we consider Eq. (35), consisting of three terms. The question is now at which time scales which terms dominate.

We start with the analysis of Eq. (35). First, both for $A_{1} \gg\left|A_{2}\right|(2 \pi k / N)^{2}$ and for $A_{1} \gg\left|A_{3}\right|(2 \pi k / N)^{4}$ one can approximate $\varphi_{k}$ well by the first term of Eq. (35). This, together with the condition $k \ll N / 2$, gives

$$
\varphi_{k} \simeq A_{1}\left(\frac{2 \pi k}{N}\right)^{2} \text { for } 1<k \ll \frac{N}{2 \pi} \min \left(\sqrt{\frac{A_{1}}{\left|A_{2}\right|}}, \sqrt[4]{\frac{A_{1}}{\left|A_{3}\right|}}, \pi\right) .
$$

This regime exists for $A_{1} \gg \max \left(4 \pi^{2}\left|A_{2}\right| / N^{2}, 16 \pi^{4}\left|A_{3}\right| / N^{4}\right)$. Second, if both $A_{2} \gg A_{1}(2 \pi k / N)^{-2}$ and $A_{2} \gg\left|A_{3}\right|(2 \pi k / N)^{2}$ hold, one has

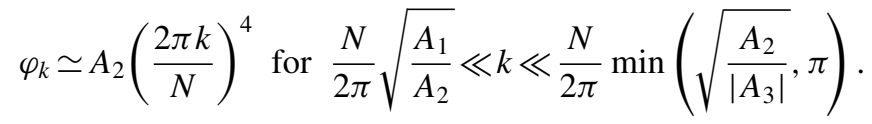

From Eq. (B2) we deduce that the regime $\varphi_{k} \sim k^{4}$ exists only for $A_{2} \gg \max \left(\sqrt{A_{1}\left|A_{3}\right|}, A_{1} / \pi^{2}\right)$. Finally, for $A_{3} \gg \max \left(A_{1}(2 \pi k / N)^{-4},\left|A_{2}\right|(2 \pi k / N)^{-2}\right)$

$\varphi_{k} \simeq A_{3}\left(\frac{2 \pi k}{N}\right)^{6}$ for $\frac{N}{2 \pi} \max \left(\sqrt{\frac{\left|A_{2}\right|}{A_{3}}}, \sqrt[4]{\frac{A_{1}}{A_{3}}}\right) \ll k \ll \frac{N}{2}$,

i.e., this regime exists for $A_{3} \gg \max \left(\left|A_{2}\right| / \pi^{2}, A_{1} / \pi^{4}\right)$.

\section{Mean-square monomer displacement $\Psi(t)$ in the limit of large $N$}

Here, we take Eqs. (B1)-(B3) into account and analyze the behavior of $\Psi(t)$, Eq. (44), for very long rings $N \gg 1$. First, at very short times, $t \ll \tau_{0} / \varphi_{N / 2}$, the use of $\lim _{x \rightarrow 0}\left[\left(1-e^{-x}\right) / x\right]=1$ leads to $\Psi(t) \simeq 2 l^{2}\left(t / \tau_{0}\right)$. On the other hand, for very long times, $t \gg \tau_{0} / \varphi_{1}$, the first term $\left(2 l^{2} / N\right)\left(t / \tau_{0}\right)$ of Eq. (44) dominates. In the intermediate time region, the motion of a monomer is subdiffusive, with respect to Eqs. (B1)-(B3). We recall that $\varphi_{k}=\varphi_{N-k}$ and consider first the regime $\varphi_{k} \sim k^{2}$, in which holds

$$
\begin{aligned}
\Psi(t) & \simeq \frac{4 l^{2}}{N} \sum_{k=1}^{k_{\max }} \frac{1-\exp \left(-\varphi_{k} t / \tau_{0}\right)}{\varphi_{k}} \\
& \approx \frac{2 l^{2}}{\pi \sqrt{A_{1}}}\left(\frac{t}{\tau_{0}}\right)^{1 / 2} \int_{0}^{\infty} \frac{1-\exp \left(-x^{2}\right)}{x^{2}} \mathrm{~d} x .
\end{aligned}
$$


In Eq. (B4), we used the substitution $x=(2 \pi k / N) \sqrt{A_{1} t / \tau_{0}}$. The integral of Eq. (B4) has as an upper limit $x \rightarrow \infty$ implying $\left(2 \pi k_{\max } / N\right) \sqrt{A_{1} t / \tau_{0}} \gg 1$, where the $k_{\max }$ is the upper limit for $k$ from Eq. (B1). Thus, employing

$$
\int_{0}^{\infty} \frac{1-\exp \left(-x^{a}\right)}{x^{a}} \mathrm{~d} x=\frac{1}{a-1} \Gamma\left(\frac{1}{a}\right),
$$

Eq. (B4) leads to

$$
\begin{gathered}
\Psi(t) \simeq \frac{2 l^{2}}{\sqrt{\pi}} A_{1}^{-1 / 2}\left(\frac{t}{\tau_{0}}\right)^{1 / 2} \text { for } \\
\frac{N^{2}}{4 A_{1} \pi^{2}} \gg \frac{t}{\tau_{0}} \gg \max \left(\frac{\left|A_{2}\right|}{A_{1}^{2}}, \sqrt{\frac{\left|A_{3}\right|}{A_{1}^{3}}}, \frac{1}{A_{1} \pi^{2}}\right) .
\end{gathered}
$$

Similarly, in the regime $\varphi_{k} \sim k^{4}$ we have

$$
\begin{aligned}
\Psi(t) & \simeq \frac{4 l^{2}}{N} \sum_{k=k_{\min }}^{k_{\max }} \frac{1-\exp \left(-\varphi_{k} t / \tau_{0}\right)}{\varphi_{k}} \\
& \approx \frac{2 l^{2}}{\pi \sqrt[4]{A_{2}}}\left(\frac{t}{\tau_{0}}\right)^{3 / 4} \int_{0}^{\infty} \frac{1-\exp \left(-x^{4}\right)}{x^{4}} \mathrm{~d} x
\end{aligned}
$$

where we used the substitution $x=(2 \pi k / N) \sqrt[4]{A_{2} t / \tau_{0}}$. Now, from Eq. (B2) $k_{\min }=(N / 2 \pi) \sqrt{A_{1} / A_{2}}$ and $k_{\max }$ $=(N / 2 \pi) \min \left(\sqrt{A_{2} /\left|A_{3}\right|}, \pi\right)$ are the lower and the upper bounds of $k$. Given that $x \in(0 ; \infty)$, the MSD of Eq. (B7) is as follows:

$$
\begin{aligned}
& \Psi(t) \simeq \frac{2 l^{2}}{3 \pi} \Gamma\left(\frac{1}{4}\right) A_{2}^{-1 / 4}\left(\frac{t}{\tau_{0}}\right)^{3 / 4} \text { for } \\
& \frac{A_{2}}{A_{1}^{2}} \gg \frac{t}{\tau_{0}} \gg \max \left(\frac{A_{3}^{2}}{A_{2}^{3}}, \frac{1}{A_{2} \pi^{4}}\right) .
\end{aligned}
$$

Finally, in the regime $\varphi_{k} \sim k^{6}$ we have

$$
\begin{aligned}
\Psi(t) & \simeq \frac{4 l^{2}}{N} \sum_{k=k_{\min }}^{[N / 2]} \frac{1-\exp \left(-\varphi_{k} t / \tau_{0}\right)}{\varphi_{k}} \\
& \approx \frac{2 l^{2}}{\pi \sqrt[6]{A_{3}}}\left(\frac{t}{\tau_{0}}\right)^{5 / 6} \int_{0}^{\infty} \frac{1-\exp \left(-x^{6}\right)}{x^{6}} \mathrm{~d} x .
\end{aligned}
$$

Here, the substitution $x=(2 \pi k / N) \sqrt[6]{A_{3} t / \tau_{0}}$ implies that $\left(2 \pi k_{\min } / N\right) \sqrt[6]{A_{3} t / \tau_{0}} \ll 1$ with $k_{\min }=(N / 2 \pi) \max$ $\left(\sqrt{\left|A_{2}\right| / A_{3}}, \sqrt[4]{A_{1} / A_{3}}\right)$. Hence, together with Eq. (B5), we obtain

$$
\begin{aligned}
\Psi(t) \simeq & \frac{2 l^{2}}{5 \pi} \Gamma\left(\frac{1}{6}\right) A_{3}^{-1 / 6}\left(\frac{t}{\tau_{0}}\right)^{5 / 6} \text { for } \\
& \min \left(\frac{A_{3}^{2}}{\left|A_{2}\right|^{3}}, \sqrt{\frac{A_{3}}{A_{1}^{3}}}\right) \gg \frac{t}{\tau_{0}} \gg \frac{1}{A_{3} \pi^{6}} .
\end{aligned}
$$

\section{Radius of gyration}

Let $\mathbf{r}_{1}, \mathbf{r}_{2}, \ldots, \mathbf{r}_{N}$ be the positions of the ring's monomers as they follow from the bonds $\mathbf{d}_{1}, \mathbf{d}_{2}$, etc. The gy- ration radius $\left\langle R_{g}^{2}\right\rangle$ is given by ${ }^{48}$

$$
\left\langle R_{g}^{2}\right\rangle \equiv \frac{1}{N} \sum_{i=1}^{N}\left\langle\left(\mathbf{r}_{i}-\mathbf{R}_{C}\right)^{2}\right\rangle
$$

where

$$
\mathbf{R}_{C} \equiv \frac{1}{N} \sum_{i=1}^{N} \mathbf{r}_{i}
$$

is the position of the center of mass. Recalling the findings of Ref. 60, as a function of the bond-bond correlations $\left\langle R_{g}^{2}\right\rangle$ reads

$$
\left\langle R_{g}^{2}\right\rangle=\frac{1}{N^{2}} \sum_{a, b=1}^{N-1} B_{a b}\left\langle\mathbf{d}_{a} \cdot \mathbf{d}_{b}\right\rangle .
$$

In Eq. (B13), the $(N-1) \times(N-1)$ matrix $\mathbf{B}=\left(B_{i j}\right)$ is given by ${ }^{60}$

$$
B_{a b}=N \min (a, b)-a b .
$$

\section{Bond-bond correlations for the nonhomogeneous semiflexible ring}

As it was shown in Eqs. (47) and (48), the bond-bond correlations in the nonhomogeneous case are given by

$$
\left\langle\mathbf{d}_{a} \cdot \mathbf{d}_{b}\right\rangle=l^{2}\left(\left(\tilde{\mathbf{W}}+\frac{1}{\epsilon^{2}} \mathbf{L}\right)^{-1}\right)_{a b} .
$$

Before starting with the calculation of the inverse matrix on the rhs of Eq. (B15), we first note that

$$
\mathbf{L}=\mathbf{e} \otimes \mathbf{e}^{T}
$$

holds, where e is a vector (column matrix) whose elements equal unity, $\mathbf{e}^{T}$ its transposed, reading $\mathbf{e}^{T}=(1, \ldots, 1)$, and $\otimes$ denotes the Kronecker (outer) product. This allows to use the Sherman-Morisson formula (see, e.g., Eq. (2.7.2) of Ref. 61)

$$
\left(\mathbf{M}+\mathbf{u} \otimes \mathbf{v}^{T}\right)^{-1}=\mathbf{M}^{-1}-\frac{\left(\mathbf{M}^{-1} \mathbf{u}\right) \otimes\left(\mathbf{v}^{T} \mathbf{M}^{-1}\right)}{1+\mathbf{v}^{T} \mathbf{M}^{-1} \mathbf{u}} .
$$

Thus,

$$
\left(\tilde{\mathbf{W}}+\frac{1}{\epsilon^{2}} \mathbf{e} \otimes \mathbf{e}^{T}\right)^{-1}=\tilde{\mathbf{W}}^{-1}-\frac{\left(\tilde{\mathbf{W}}^{-1} \mathbf{e}\right) \otimes\left(\mathbf{e}^{T} \tilde{\mathbf{W}}^{-1}\right)}{\epsilon^{2}+\mathbf{e}^{T} \tilde{\mathbf{W}}^{-1} \mathbf{e}} .
$$

Inserting Eq. (B18) into Eq. (B15) and taking the limit $\epsilon \rightarrow 0$ we obtain

$$
\left\langle\mathbf{d}_{a} \cdot \mathbf{d}_{b}\right\rangle=l^{2}\left[\left(\tilde{\mathbf{W}}^{-1}\right)_{a b}-\frac{\sum_{k}\left(\tilde{\mathbf{W}}^{-1}\right)_{a k} \sum_{n}\left(\tilde{\mathbf{W}}^{-1}\right)_{n b}}{\sum_{n, k}\left(\widetilde{\mathbf{W}}^{-1}\right)_{n k}}\right] .
$$

We note that Eq. (B19) can also be obtained by considering general Gaussian distributions under strict (holonomic) constraints, ${ }^{36}$ fact recently used in the treatment of nonMarkovian diffusion-limited reactions. ${ }^{37-39}$

We recall that the matrix $\widetilde{\mathbf{W}}=\left(\widetilde{W}_{a b}\right)$ describes the potential energy of a chain with stiffness parameters $\widetilde{q}_{1}$ and $\widetilde{q}_{2}$, see Eq. (4). The elements of its inverse, $\widetilde{\mathbf{W}}^{-1}$, are known ${ }^{28}$ and given by

$$
\left(\widetilde{\mathbf{W}}^{-1}\right)_{a b}=\frac{1}{2 \sigma}\left(c_{+} b_{+}^{|a-b|}-c_{-} b_{-}^{|a-b|}\right) .
$$


In Eq. (B20),

$$
\begin{gathered}
c_{ \pm}=(1+\widetilde{p}) \widetilde{q}_{1} \pm \sigma, \\
b_{ \pm}=\left[(1-\widetilde{p}) \widetilde{q}_{1} \pm \sigma\right] / 2,
\end{gathered}
$$

and

$$
\sigma=\sqrt{(1-\widetilde{p})^{2} \widetilde{q}_{1}^{2}+4 \widetilde{p}}
$$

where $\widetilde{p}=\left(\widetilde{q}_{2}-\widetilde{q}_{1}^{2}\right) /\left(1-\widetilde{q}_{1}^{2}\right)$. With Eq. (B20) the sums in Eq. (B19) can be readily calculated using the geometric series and the algebraic relations

$$
\frac{1}{2 \sigma}\left(c_{+} \frac{1+b_{+}}{1-b_{+}}-c_{-} \frac{1+b_{-}}{1-b_{-}}\right)=\frac{\left(1+\widetilde{q}_{1}\right)(1+\widetilde{p})}{\left(1-\widetilde{q}_{1}\right)(1-\widetilde{p})}
$$

and

$\frac{1}{2 \sigma}\left(\frac{c_{+} b_{+}}{\left(1-b_{+}\right)^{2}}-\frac{c_{-} b_{-}}{\left(1-b_{-}\right)^{2}}\right)=\frac{\widetilde{q}_{1}\left(1+\widetilde{p}^{2}\right)+2 \widetilde{p}}{\left(1-\widetilde{q}_{1}\right)^{2}(1-\widetilde{p})^{2}}$.

In Eqs. (B24) and (B25), the $c_{ \pm}, b_{ \pm}$, and $\sigma$ are of Eqs. (B21)(B23). Hence, the sums in Eq. (B19) are

$$
\begin{aligned}
\sum_{k=1}^{N}\left(\widetilde{\mathbf{W}}^{-1}\right)_{a k}= & \frac{\left(1+\widetilde{q}_{1}\right)(1+\widetilde{p})}{\left(1-\widetilde{q}_{1}\right)(1-\tilde{p})} \\
& -\frac{1}{2 \sigma}\left(c_{+} \frac{b_{+}^{a}+b_{+}^{N-a+1}}{1-b_{+}}-c_{-} \frac{b_{-}^{a}+b_{-}^{N-a+1}}{1-b_{-}}\right)
\end{aligned}
$$

and

$$
\begin{aligned}
\sum_{k, n=1}^{N}\left(\widetilde{\mathbf{W}}^{-1}\right)_{n k}= & N \frac{\left(1+\widetilde{q}_{1}\right)(1+\widetilde{p})}{\left(1-\widetilde{q}_{1}\right)(1-\widetilde{p})}-2 \frac{\widetilde{q}_{1}\left(1+\widetilde{p}^{2}\right)+2 \widetilde{p}}{\left(1-\widetilde{q}_{1}\right)^{2}(1-\widetilde{p})^{2}} \\
& +\frac{1}{\sigma}\left(\frac{c_{+} b_{+}^{N+1}}{\left(1-b_{+}\right)^{2}}-\frac{c_{-} b_{-}^{N+1}}{\left(1-b_{-}\right)^{2}}\right) \cdot \quad
\end{aligned}
$$

Thus, the correlations between the bonds $\left\{\mathbf{d}_{a}\right\}$ of a nonhomogeneous ring are determined through Eq. (B19), under the use of Eqs. (B20), (B26), and (B27).

\section{APPENDIX C: SPECIAL CASES: THE HOMOGENEOUS RING UNDER FREELY ROTATING AND UNDER FULLY FLEXIBLE CONDITIONS}

In this appendix, we show that the model of Sec. III reproduces, both in the limit of vanishing DR, i.e., of freely rotating bonds as well as in the limit of fully flexible rings, results known from the literature.

\section{Freely rotating limit}

The freely rotating limit is obtained by setting $\gamma=0$ in Eq. (6). Then from Eq. (17) one has

$$
\left\langle\mathbf{d}_{a} \cdot \mathbf{d}_{b}\right\rangle=\frac{l^{2}}{N} \sum_{k=1}^{N-1} \frac{\cos [2 \pi k(a-b) / N]}{\alpha+2 \beta \cos \left(\frac{2 \pi k}{N}\right)} .
$$

Furthermore, by also using Eq. (19),

$$
q_{1}=\sum_{k=1}^{N-1} \frac{x_{k}}{x-x_{k}} / \sum_{k=1}^{N-1} \frac{1}{x-x_{k}},
$$

where we have set $x \equiv-\alpha /(2 \beta)$ and

$$
x_{k}=\cos \left(\frac{2 \pi k}{N}\right) \text {. }
$$

In Ref. 33, it was shown that the $x$ defined as in Eq. (C2) obeys

$$
\frac{x-q_{1}}{N-1} \frac{1}{\sigma(x)} \frac{\mathrm{d} \sigma(x)}{\mathrm{d} x}-1=0,
$$

where one has

$$
\sigma(x)=\frac{N\left(T_{N}(x)-1\right)}{x-1},
$$

$T_{N}(x)$ being the Chebyshev polynomial of the first kind of degree $N$. For $x>1$, considering a discrete wormlike ring, Berg $^{57}$ has obtained an equation similar to Eq. (C4), but with different numerical coefficients. ${ }^{33,57}$ The continuous version of Eq. (C4) leads to the result of Ref. 5 As we proceed to show, Eqs. (C2) and (C4) are equivalent, thus they lead to the same $x$.

Now, the roots of the numerator of Eq. (C5), $N\left(T_{N}(x)-1\right)$, are the $x_{k}$ of Eq. (C3) with $k=1, \ldots, N$, see Ref. 62. Hence,

$$
\sigma(x)=N C \prod_{k=1}^{N-1}\left(x-x_{k}\right),
$$

where $C$ is some constant and

$$
\frac{1}{\sigma(x)} \frac{\mathrm{d} \sigma(x)}{\mathrm{d} x}=\sum_{k=1}^{N-1} \frac{1}{x-x_{k}} .
$$

Substituting Eq. (C7) into Eq. (C4), Eq. (C2) follows.

The eigenvalue spectra of the rings with freely rotating bonds $\left\{\varphi_{k}^{F R}\right\}$ are straightforwardly obtained from $\left\{\varphi_{k}\right\}$ of Eq. (34), by setting $\gamma=0$

$$
\varphi_{k}^{F R}=(\alpha+2 \beta) \xi_{k}-\beta \xi_{k}^{2} .
$$

Using Eqs. (18)-(20) and (33) and that $x \equiv-\alpha /(2 \beta)$ we obtain

$$
\varphi_{k}^{F R}=\frac{N-1}{N}\left[\frac{8}{x-q_{1}} \sin ^{4}\left(\frac{\pi k}{N}\right)+\frac{4(x-1)}{x-q_{1}} \sin ^{2}\left(\frac{\pi k}{N}\right)\right],
$$

where $x$ is as in Eq. (C2) and $k$ runs from 1 to $N$. Given that Eqs. (C2) and (C4) have the same solution for $x$, Eq. (C9) leads to the eigenvalues given by Eq. (71) of Ref. 33

\section{Fully flexible limit}

The fully flexible limit is obtained by neglecting all the constraints arising from the $\mathrm{NN}$ and the NNN bonds, Eqs. (19) and (20), i.e., by taking $\beta=0$ and $\gamma=0$. From Eq. (18) it follows that

$$
\alpha=\frac{N-1}{N} .
$$


Using that

$$
\sum_{k=1}^{N-1} \cos \left(\frac{2 \pi k}{N}\right)=-1
$$

Eq. (17) gives

$$
\frac{\left\langle\mathbf{d}_{i} \cdot \mathbf{d}_{j}\right\rangle}{l^{2}}= \begin{cases}1 & \text { for } i=j \\ -1 /(N-1) & \text { for } i \neq j,\end{cases}
$$

in line with previous works. ${ }^{34,59}$

${ }^{1}$ M. Bixon and R. Zwanzig, J. Chem. Phys. 68, 1896 (1978),

${ }^{2}$ A. Perico and M. Guenza, J. Chem. Phys. 84, 510 (1986).

${ }^{3}$ M. Guenza and A. Perico, Macromolecules 25, 5942 (1992).

${ }^{4}$ R. G. Winkler, P. Reineker, and L. Harnau, J. Chem. Phys. 101, 8119 (1994).

${ }^{5}$ B.-Y. Ha and D. Thirumalai, J. Chem. Phys. 103, 9408 (1995).

${ }^{6}$ L. Harnau, R. G. Winkler, and P. Reineker, J. Chem. Phys. 106, 2469 (1997).

${ }^{7}$ J. J. Portman, S. Takada, and P. G. Wolynes, J. Chem. Phys. 114, 5069 (2001).

${ }^{8}$ M. G. Guenza, J. Phys.: Condens. Matter 20, 033101 (2008).

${ }^{9}$ M. Dolgushev and A. Blumen, J. Chem. Phys. 131, 044905 (2009).

${ }^{10}$ R. G. Winkler, J. Chem. Phys. 133, 164905 (2010).

${ }^{11}$ G. T. Barkema and J. M. J. van Leeuwen, J. Stat. Mech. 2012, P12019.

${ }^{12}$ A. Kumar, G. J. Rai, and P. Biswas, J. Chem. Phys. 138, 104902 (2013).

${ }^{13}$ R. D. Groot, J. Chem. Phys. 138, 224903 (2013).

${ }^{14}$ D. T. Seaton, S. Schnabel, D. P. Landau, and M. Bachmann, Phys. Rev. Lett. 110, 028103 (2013).

${ }^{15}$ Y. Jiang and J. Z. Y. Chen, Phys. Rev. Lett. 110, 138305 (2013).

${ }^{16}$ C. Bustamante, J. F. Marco, E. D. Siggia, and S. Smith, Science 265, 1599 (1994).

${ }^{17}$ J. Käs, H. Strey, J. X. Tang, D. Finger, R. Ezzel, E. Sackmann, and P. A. Janmey, Biophys. J. 70, 609 (1996).

${ }^{18}$ R. Götter, K. Kroy, E. Frey, M. Bärmann, and E. Sackmann, Macromolecules 29, 30 (1996).

${ }^{19}$ M. Dolgushev and A. Blumen, J. Chem. Phys. 132, 124905 (2010).

${ }^{20}$ M. Dolgushev, G. Berezovska, and A. Blumen, J. Chem. Phys. 133, 154905 (2010).

${ }^{21}$ M. Dolgushev, G. Berezovska, and A. Blumen, Macromol. Theory Simul. 20, 621 (2011).

${ }^{22}$ F. Fürstenberg, M. Dolgushev, and A. Blumen, J. Chem. Phys. 136, 154904 (2012).

${ }^{23}$ F. Fürstenberg, M. Dolgushev, and A. Blumen, J. Chem. Phys. 138, 034904 (2013).

${ }^{24}$ A. A. Gurtovenko and A. Blumen, Adv. Polym. Sci. 182, 171 (2005).

${ }^{25}$ P. J. Flory, Statistical Mechanics of Chain Molecules (Oxford University Press, New York, 1988).

${ }^{26}$ A. Korkut and W. A. Hendrickson, Proc. Natl. Acad. Sci. U.S.A. 106, 15667 (2009).

${ }^{27}$ A. Altis, M. Otten, P. H. Nguyen, R. Hegger, and G. Stock, J. Chem. Phys. 128, 245102 (2008).
${ }^{28}$ M. Dolgushev and A. Blumen, J. Chem. Phys. 138, 204902 (2013).

${ }^{29}$ M. Trabi and D. J. Craik, Trends Biochem. Sci. 27, 132 (2002).

${ }^{30}$ K. Alim and E. Frey, Phys. Rev. Lett. 99, 198102 (2007).

${ }^{31}$ Z. Benková and P. Cifra, Macromolecules 45, 2597 (2012).

${ }^{32}$ W. Chen, J. Chen, L. Liu, X. Xu, and L. An, Macromolecules 46, 7542 (2013).

${ }^{33}$ M. Dolgushev, G. Berezovska, and A. Blumen, J. Chem. Phys. 135, 094901 (2011).

${ }^{34}$ A. Y. Grosberg, Macromolecules 41, 4524 (2008).

${ }^{35}$ L. D. Landau and E. M. Lifshitz, Mechanics (Pergamon Press, Oxford, 1969).

${ }^{36}$ M. L. Eaton, Multivariate Statistics: A Vector Space Approach (Institute of Mathematical Statistics, Beachwood, OH, 1983), Vol. 53.

${ }^{37}$ T. Guérin, O. Bénichou, and R. Voituriez, Nat. Chem. 4, 568 (2012).

${ }^{38}$ T. Guérin, O. Bénichou, and R. Voituriez, J. Chem. Phys. 138, 094908 (2013).

${ }^{39}$ T. Guérin, O. Bénichou, and R. Voituriez, Phys. Rev. E 87, 032601 (2013).

${ }^{40} \mathrm{G}$. Strobl, The Physics of Polymers (Springer, Berlin, 1996).

${ }^{41}$ G. Witz, K. Rechendorff, J. Adamcik, and G. Dietler, Phys. Rev. Lett. 101, 148103 (2008)

${ }^{42}$ D. Kawaguchi, Polym. J. 45, 783 (2013).

${ }^{43}$ F. Amblard, A. C. Maggs, B. Yurke, A. N. Pergellis, and S. Leibler, Phys. Rev. Lett. 77, 4470 (1996)

${ }^{44}$ E. T. Jaynes, Phys. Rev. 106, 620 (1957).

${ }^{45}$ H. Haken, Synergetik (Springer, Berlin, 1983).

${ }^{46}$ J. M. Ziman, Principles of the Theory of Solids (Cambridge University Press, London, 1972).

${ }^{47}$ See supplementary material at http://dx.doi.org/10.1063/1.4885445 for the detailed calculations.

${ }^{48}$ M. Doi and S. F. Edwards, The Theory of Polymer Dynamics (Clarendon Press, Oxford, 1986).

${ }^{49}$ A. Y. Grosberg and A. R. Khokhlov, Statistical Physics of Macromolecules (American Institute of Physics, New York, 1994).

${ }^{50}$ N. Biggs, Algebraic Graph Theory, 2nd ed. (Cambridge University Press, Cambridge, 1993).

${ }^{51}$ V. Bloomfield and B. H. Zimm, J. Chem. Phys. 44, 315 (1966).

${ }^{52}$ A. J. Chompff, J. Chem. Phys. 53, 1566 (1970).

${ }^{53}$ C. Satmarel, A. A. Gurtovenko, and A. Blumen, Macromolecules 36, 486 (2003).

${ }^{54}$ G. Tsolou, N. Stratikis, C. Baig, P. S. Stephanou, and V. G. Mavrantzas, Macromolecules 43, 10692 (2010).

${ }^{55}$ H. Schiessel, Phys. Rev. E 57, 5775 (1998).

${ }^{56}$ P. Biswas, R. Kant, and A. Blumen, Macromol. Theory Simul. 9, 56 (2000).

${ }^{57}$ O. G. Berg, Biopolymers 18, 2861 (1979).

${ }^{58}$ B. H. Zimm and W. H. Stockmayer, J. Chem. Phys. 17, 1301 (1949).

${ }^{59}$ A. Baumgärtner, J. Chem. Phys. 76, 4275 (1982).

${ }^{60}$ W. C. Forsman and R. E. Hughes, J. Chem. Phys. 38, 2118 (1963).

${ }^{61}$ W. H. Press, S. A. Teukolski, W. T. Vetterling, and B. P. Flannery, Numerical Recipes in Fortran 77, 2nd ed. (Cambridge University Press, New York, 2003).

${ }^{62}$ J. C. Mason and D. C. Handscomb, Chebyshev Polynomials (Chapman and Hall, 2003). 